\title{
Angelika Storrer
}

\section{Hypermedia-Wörterbücher: Perspektiven für eine neue Generation elektronischer Wörterbücher}

$\begin{array}{llll}1 & \text { Familienspaß mit Wörterbüchern - alte und } & 4 & \text { Wörterbücher in Aktion } \\ & \text { neue Visionen } & 4.1 & \text { Interaktive Wörterbücher } \\ 2 & \text { Multimediale Wörterbücher: Integration von } & 4.2 & \text { Kollaborative Wörterbucherstellung im WWW } \\ & \text { Text, Bild, Ton und Video } & 4.3 & \text { Die Verbindung von CD-ROM und Internet } \\ 3 & \text { Hypertext-Wörterbücher: Suchen und Stöbern } & 5 & \text { Ausblick } \\ & \text { im lexikalischen Informationsraum } & 6 & \text { Literatur }\end{array}$

\section{Familienspaß mit Wörterbüchern - alte und neue Visionen}

In der Vorrede zu seinem „Deutschen Wörterbuch“ entwarf Jacob Grimm 1854 folgende Vision von der Benutzung seines Wörterbuchs:

\begin{abstract}
„Fände bei den Leuten die einfache Kost der heimischen Sprache eingang, so könnte das Wörterbuch zum Hausbedarf, und mit Verlangen, oft mit Andacht gelesen werden. Warum sollte sich nicht der Vater ein paar Wörter ausheben und sie abends mit den Knaben durchgehend zugleich ihre Sprachgabe prüfen und die eigne anfrischen? Die Mutter würde gern zuhören. Frauen, mit ihrem gesunden Mutterwitz und im Gedächtnis gute Sprüche bewahrend, tragen oft wahre Begierde ihr unverdorbenes Sprachgefühl zu üben, vor die Kisten und Kasten zu treten, aus denen wie gefaltete Leinwand lautere Wörter ihnen entgegen quellen: Ein Wort, ein Reim führt dann auf andere und sie kehren öfter zurück und heben den Deckel von neuem.“ (Grimm 1854, XIIf.)
\end{abstract}

Diese Zitatstelle wurde in der Nachfolge häufig belächelt und dies nicht etwa wegen Grimms positiver Einschätzung der weiblichen Sprachbegabung. Vielmehr paßt das Bild einer um das Grimmsche Wörterbuch versammelten, in spielerische Sprachreflexion versunkenen Familie nicht recht zur gängigen Vorstellung vom Wörterbuch als Gebrauchsgegenstand, der als Referenzwerk zur Lösung spezifischer Probleme bei der Sprachproduktion und -rezeption herangezogen wird, und von daher eher am Arbeitsplatz von Menschen aufzufinden ist, die auf die eine oder andere Art beruflich mit Sprache zu tun haben. Nicht zufällig verwenden Benutzeroberflächen moderner elektronischer Wörterbücher Metaphern wie Schreibtisch, Zettelkasten, Karteikasten; nicht zufällig stand hinter der Computerisierung von Wörterbüchern bislang vornehmlich der Wunsch nach mehr Schnelligkeit beim Zugriff, besseren und flexibleren Suchmöglichkeiten durch entsprechende Retrievalwerkzeuge und möglichst komfortablen Verknüpfungen mit anderen zur Arbeit benötigten Programmsystemen wie Textverarbeitungsprogrammen oder Systemen zur computerunterstützten Übersetzung. 
Die bunte Welt von Hypermedia, einer Zusammensetzung aus Hypertext und Multimedia, läßt nun auch die Idee des Wörterbuchs für die ganze Familie wieder aufleben. So begeisterte sich Florian Langenscheidt anläßlich einer Pressekonferenz auf der Frankfurter Buchmesse 1994, auf der u.a. die Hypermedia-Anwendung „Mein erstes Lexikon“ vorgestellt wurde:

„Elektronische Bücher bei DUDEN, Meyer und Brockhaus - das waren bis jetzt Nachschlagewerke für den Arbeitsplatz am PC. Aber auch vor den Privathaushalten und vor dem Familienleben machen elektronische Bücher nicht halt. Hier bringen sie völlig neue Dimensionen der „gemeinsamen Lektüre“ ins Spiel. Und spielerische unterhaltsame Multimedia-Titel werden dann auch im Frühjahr nächsten Jahres zu den Highlights unserer Novitäten werden! Erwachsene und Kinder werden begeistert sein von den sensationellen Produkten, die in Zusammenarbeit mit dem führenden Multimediaverlag Dorling Kindersley entstanden.“ (Informationen für die Presse, verteilt auf der Frankfurter Buchmesse am 7.10.1994)

Der Grimmsche Gedanke, Sprache über ein Wörterbuch in den Mittelpunkt familiärer Geselligkeit zu stellen, lebt also wieder auf in moderner Gestalt als sog. Edutainment- und Infotainment-Anwendungen, ${ }^{1}$ d.h. Computerprogrammen, die auf unterhaltsame Art und Weise bilden und informieren möchten. Allerdings dürfte manch zeitgenössischer Vater auf die computertechnische Nachhilfe der Tochter oder des Sohnes angewiesen sein, um die Freuden des Edutainment wirklich auskosten zu können.

Was macht Hypermedia attraktiv für Autoren und Benutzer von Nachschlagewerken? Es sind im wesentlichen die folgenden Eigenschaften, durch die Hypermedia-Wörterbücher einen Mehrwert gegenüber Print-Wörterbüchern einerseits und nicht-hypermedialen lexikalischen Datenbanken andererseits aufweisen:

1) Multimedia: In Hypermedia-Anwendung können Text, (animierte) Graphik, Ton- und Videodokumente miteinander verknüpft werden. Lexikalisches Wissen kann also unter Nutzung verschiedener Symbolsysteme repräsentiert und über verschiedene Sinneskanäle vermittelt werden. Diese Erweiterung der Ausdruckmöglichkeiten in der Lexikographie ist Thema von Abschnitt zwei.

2) Hypertext: Hypermedia-Anwendungen haben die Struktur von Netzwerken, deren Knoten aus Text-, Bild-, Ton- und Video-Objekten aufgebaut sind, und deren Kanten als Verknüpfungen (Hyperlinks) bezeichnet werden. In solchen Netzwerken können Beziehungen zwischen lexikalischen Einheiten direkt als Verknüpfungen abgebildet werden, die durch einen einfachen Mausklick zu thematisch relationierten Knoten führen. Während lexikalische Datenbanken den Zugriff auf die lexikalischen Einheiten nach dem sog. „Pattern-Matching-Paradigma“ organisieren, bei dem das System alle Einheiten anzeigt, die einem vom Benutzer spezifizierten Suchmuster entsprechen, unterstützen Hypertext-Systeme zusätzlich die Informationserschließung nach dem das sog. „explorativen Paradigma“, dem Durchstöbern des Wörterbuchs im Zuge eines nicht präzise formulierbaren Informationsbedarfs durch die Verfolgung der im Wörterbuch angelegten Verknüpfungen. Dieser für das Wiederaufleben des Lesebuchgedankens zentrale Aspekt wird in Abschnitt 3 ausgeführt werden.

3) Interaktivität: Mit dem Schlagwort „interaktiv“" wird die Möglichkeit des Benutzers bezeichnet, das Verhalten einer Softwareumgebung zu beeinflußen und dieses an seine individuellen Bedürfnisse anzupassen. So können in Hypermedia-Wörterbüchern eigene Verknüpfungen angelegt, Anmerkungen hinzugefügt und Teilbereiche der Wortschatzbeschreibungen unter bestimmten Aspekten neu organisiert werden. Einige Formen der Benutzer-System-Interaktion in Hypermedia-Wörterbüchern werden in Abschnitt 4.1 vorgestellt. Neue Formen der computerunterstützten Benutzer-Benutzer-Interaktion entwickeln sich im Internet, einem weltweitem Verbund von Computernetzen. Mit sog. „Browsern“ können auf einfache Weise sowohl Informationsdienste wie das hypertextorientierte World Wide Web als auch kommunikationsorientierte Dienste wie elektronische Post und Diskussionsforen genutzt werden. In Abschnitt 4.2 möchte ich an einigen Beispielen zeigen, wie durch die Verbindung von Informations- und Kommunikationsdiensten neue kollaborative Formen der Wörterbucharbeit entstehen. Welche Vorteile und Chancen diese Publikations-

1 Es handelt sich um Zusammenziehungen aus Education und Entertainment bzw. Information und Entertainment. 
form für die wissenschaftliche Fachlexikographie bietet, beschreiben Lemberg/Petzold et al. (im selben Band).

Der vorliegende Aufsatz gibt keinen vollständigen Überblick über aktuelle Hypermedia-Anwendungen in der Lexikographie. Vielmehr möchte ich an geeigneten Beispielen die Mehrwerte der hypermedialen Darstellungsform gegenüber dem gedruckten Medium herausarbeiten und zeigen, wie gedruckte Wörterbücher aufbereitet werden müssen, damit das Potential von Hypermedia auch wirklich ausgeschöpft werden kann. Die Wertungen der HypermediaWörterbücher beziehen sich deshalb auch nur auf den Aspekt der medialen Umsetzung und sollten nicht als Rezensionen mißverstanden werden. Für eine Rezension elektronischer Wörterbücher spielen Gesichtspunkte der lexikographischen Bearbeitung und der inneren und äußeren Selektion eine tragende Rolle, entsprechend schneiden Anwendungen traditioneller Wörterbuchverlage meist sehr gut ab, auch wenn die Möglichkeiten von Hypermedia noch wenig genutzt werden. ${ }^{2}$

\section{Multimediale Wörterbücher: Integration von Text, Bild, Ton und Video}

Als „Multimedia“ wird die Integration statischer Medien wie Text, Graphik, und dynamischer Medien wie Animation, gesprochene Sprache, Musik und Video in einer Softwareanwendung bezeichnet. ${ }^{3}$ Multimedia bringt Farbe, Musik und Bewegung in das ehemals textdominierte Wörterbuch und ist sicherlich zunächst der Aspekt, der Hypermedia-Wörterbücher auch für Menschen attraktiv macht, die sonst nur selten ein gedrucktes Wörterbuch aufschlagen würden.

Multimedia spielt eine Rolle sowohl bei der Ausgestaltung der Benutzerschnittstelle als auch bei der lexikographischen Sprachbeschreibung. Multimediale Benutzeroberflächen dienen dazu, elektronische Wörterbücher attraktiv zu verpacken und ihre Bedienbarkeit zu vereinfachen. Die Funktionalität des System, d.h. die Zugriffs- und Interaktionsmöglichkeiten werden dem Benutzer häufig über Metaphern vermittelt, die an den Umgang mit vertrauten Medien anknüpfen. Inwieweit dies wirklich zu einer „intuitiv" bedienbaren Benutzerschnittstelle führt, hängt davon ab, wie gut sich die Funktionalität des Systems auf einen Metaphernbereich abbilden läßt. Sollen verschiedene Nachschlagewerke mit derselben Oberfläche verwaltet werden, bieten sich Bibliotheken und Bücherregale als Ausgangsbereiche an, aus denen „Bücher“ ausgewählt und auf virtuelle Schreibtische und Arbeitsflächen „gelegt“ werden können. Beispiele hierfür sind das Bee-Book-System, mit dem Nachschlagewerke von Bertelsmann verwaltet werden, und die PC-Bibliothek, in die Nachschlagewerke des Bibliographischen Instituts und von Langenscheidt eingestellt werden können (Storrer 1996). Die Metaphern vereinfachen die Formulierung der Metatexte zur Systembenutzung und sie bestimmen die Ausgestaltung der graphischen Benutzeroberfläche durch entsprechende Ikonen (Regal, Buch, Karteikarte), die per Mausklick aktiviert werden können. Getreu dem Ratschlag „build magic into your applica-

2 Lesenswerte Rezensionen sind z.B. Leckebusch (1995) und Neth/Müller (1997).

3 Einen aktuellen Überblick über die verschiedene Definitionen für Multimedia gibt Schulmeister $(1996,15-$ - 20). Weidenmann $(1995,65 f f$.) differenziert den Ausdruck „Multimedia“ weiter in den Dimensionen Medium, Kodierung und Sinnesmodalität. 
tion“ (Gloor 1990, 49) können als anregende Zusätze kleine Toneinlagen hinzukommen, z.B. ist in Microsoft Bookshelf (Bookshelf 1995) die Aktion „Öffnen eines neuen Buches“ mit einem Seitenblätter-Geräusch verbunden. Gloor warnt jedoch zurecht vor zuviel inhaltlich leerem Multimedia-Zauber, der anfängliche Amüsiertheit schnell in Überdruß und Genervtheit umschlagen läßt; ein Negativ-Beispiel sind die lärmigen Musik- und Videoeinlagen, die beim Systemstart von Langenscheidt's Language Explorer, im weiteren LLE (1997) (s.u.) abgespielt werden.

Multimedia bereichert aber vor allem die Möglichkeiten lexikographischer Sprachbeschreibung. Hypermedia-Lexikographen können verschiedene Symbolsysteme nutzen und unterschiedliche Sinnesmodalitäten ansprechen; Bild-, Ton- und Videoobjekte ergänzen das bislang textdominierte Wörterbuch aufs beste. Der erste Schritt zur multimedialen Aufbereitung allgemeinsprachlicher Wörterbücher besteht meist darin, die über phonetische Umschriften vermittelten Ausspracheangaben um Audiodateien mit Aussprache und Betonung zu ergänzen. Eine solche Ergänzung ist vor allem für mehrsprachige Wörterbücher attraktiv und für Wörterbuchbenutzer, die phonetische Umschriften nicht oder nur schwer lesen können. Entsprechend wurde in der Rezension von Neth/Müller (1997) das Vorhandensein solcher Ausspracheangaben auch als Qualitätsmerkmal von Hypermedia-Wörterbüchern gewertet.

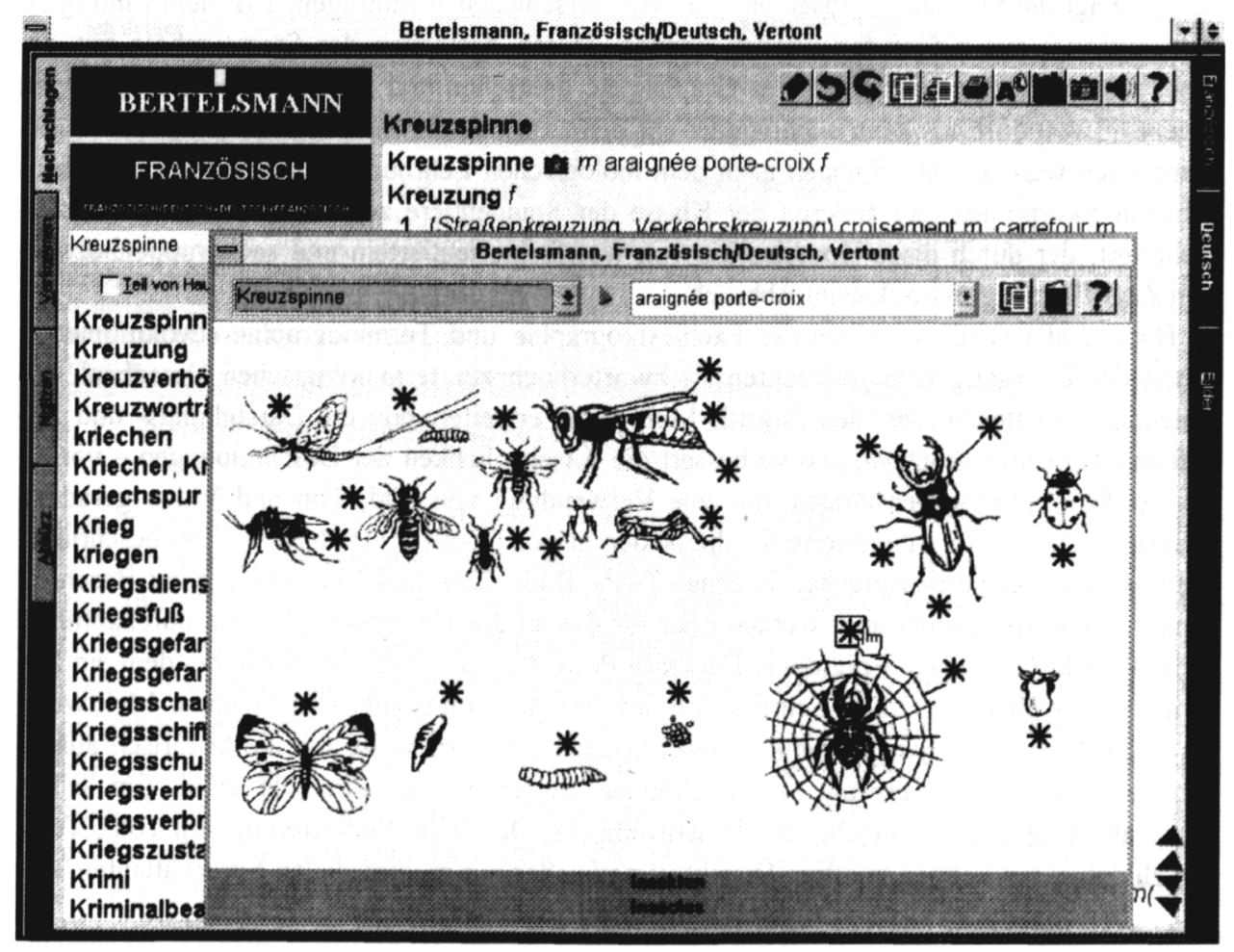

Abb. 1: Bildersammlung „Insekten - les insectes“ aus dem TWF

Bilder und Videosequenzen werden benutzt, um Bedeutungserläuterungen für Konkreta im Bereich der Nennlexik zu illustrieren. Vor allem nicht-muttersprachliche Wörterbuchbenutzer 
freuen sich sicher über Fotos oder Graphiken, die im semantischen Kommentar des entsprechenden Wörterbuchartikels die sprachlichen Bedeutungserläuterungen ergänzen. Aber auch Lexikographen, die Wörter wie Tapir oder Stele bearbeiten, werden sich wohler fühlen, wenn sie der rein sprachlichen Bedeutungsparaphrasenangabe - im Duden Universalwörterbuch $\mathrm{DUW}^{4}$ für Tapir „plumpes Säugetier in den tropischen Wäldern Amerikas u. Asiens mit kurzem, dichtem Fell u. einem kurzen Rüssel“ - ein Bild hinzufügen können. Dies wird es Wörterbuchbenutzern erleichtert, Tapire als solche zu erkennen und korrekt zu benennen. Noch besser, wenn neben Bildern auch Musik und Videosequenzen eingebunden werden können. Im Wörterbucheintrag der Microsoft Bookshelf zu Flamenco läßt sich beispielsweise ein Video abrufen, das nicht nur die für den Tanz typischen Bewegungen, sondern auch den Rhythmus der dazugehörigen Musik vermittelt.

Bilder und Videos können nicht nur die Bedeutungsparaphrasenangabe für ein Einzelwort ergänzen; sie können auch den sachgruppenorientierten Zugriff auf Wörterbucheinträge unterstützen. In dem vom Bee-Book-System verwalteten Taschenwörterbuch französisch-deutsch deutsch-französisch, im weiteren als TWF abgekürzt (Bee-Book (TWF) 1996), sind beispielsweise Illustrationen von zusammengehörigen Objekten mit den zugehörigen Wörterbucheinträgen verknüpft - der Verpackungstext spricht von einem „,integrierten Bildwörterbuch“. Abbildung 1 zeigt die Sammlung „Insekten“, die von verschiedenen Einträgen, z.B. dem Eintrag zu Kreuzspinne, aus aufgerufen werden können. Durch Anklicken der Sternsymbole mit der Maus werden in der Leiste über der Graphik die deutschen und französischen Benennungen angezeigt, von dort aus kann auch wieder auf den entsprechenden Wörterbucheintrag zurückgesprungen werden. Daß Spinnen nach dem biologischen Lehrbuch keine Insekten sind, sondern mit Skorpionen und anderen der Klasse der Spinnentiere zugeordnet werden, tut dem Mehrwert, der durch diese Kombination von wortformorientiertem und sachgruppenbezogenem Zugriff erzeugt wird, keinen Abbruch.

Hypermedia wird vor allem die Fachlexikographie und Terminographie revolutionieren. Schon der Übergang vom gedruckten Fachwörterbuch zur terminologischen Datenbank beschleunigt und flexibilisiert den Zugriff. Multimedia erweitert nun die Darstellungs- und Zugriffsmöglichkeiten erheblich und verbessert die Anschaulichkeit der Beschreibungen - vor allem bei Wörterbuchgegenständen, die eine Verwendung von Bild, Ton und Video geradezu verlangen. Dies gilt beispielsweise für die beiden in Schulmeister (1996, 288-299) beschriebenen Lexika zur Gebärdensprache, ${ }^{5}$ in denen Text-, Bild-, Ton- und Videoobjekte zur Beschreibung von Gebärden eingesetzt werden oder für das an der Universität Virginia durchgeführte MED-Projekt (= „Mayan Epigraphic Database Project“, vgl. Alvarado 1996), bei dem ein Katalog mit Glyphen der Maya-Schriftkultur im Internet aufgebaut wird. Welche Mehrwerte durch die Einbindung von Graphiken und Faksimiles beispielsweise für die historisch ausgerichtete Lexikographie erzeugt werden können, zeigen Lemberg/Petzold et al. (im selben Band) am Beispiel des deutschen Rechtswörterbuchs. Durch die Verknüpfung von Ton-, Textund Bildobjekten können künftige Dialektwörterbücher mit multimedialen Sprachatlanten ver-

4 Ich beziehe mich künftig mit dem Kürzel DUW immer auf die vom PC-Bibliothekssystem verwaltete elektronische Versison des Duden Universalwörterbuch (PC-Bib (DUW/DOG) 1989/1990).

5 Es handelt sich um das „Lexikon für Computerbegriffe mit Gebärden“ (Schulmeister 1993) und das didaktisch orientierte Wörterbuch American Sign Language (ASL)-Dictionary, das auch in einer WWW-Version (ASL) zugänglich ist. 
bunden und um authentische Sprechproben angereichert werden, wie sie beispielsweise am Spracharchiv des IDS gesammelt und in digitalisierter Form zur Verfügung gestellt werden.

Die Integration von Text, Bild, Ton und Video wird auch den Bereich der Lernerwörterbücher grundlegend verändern.

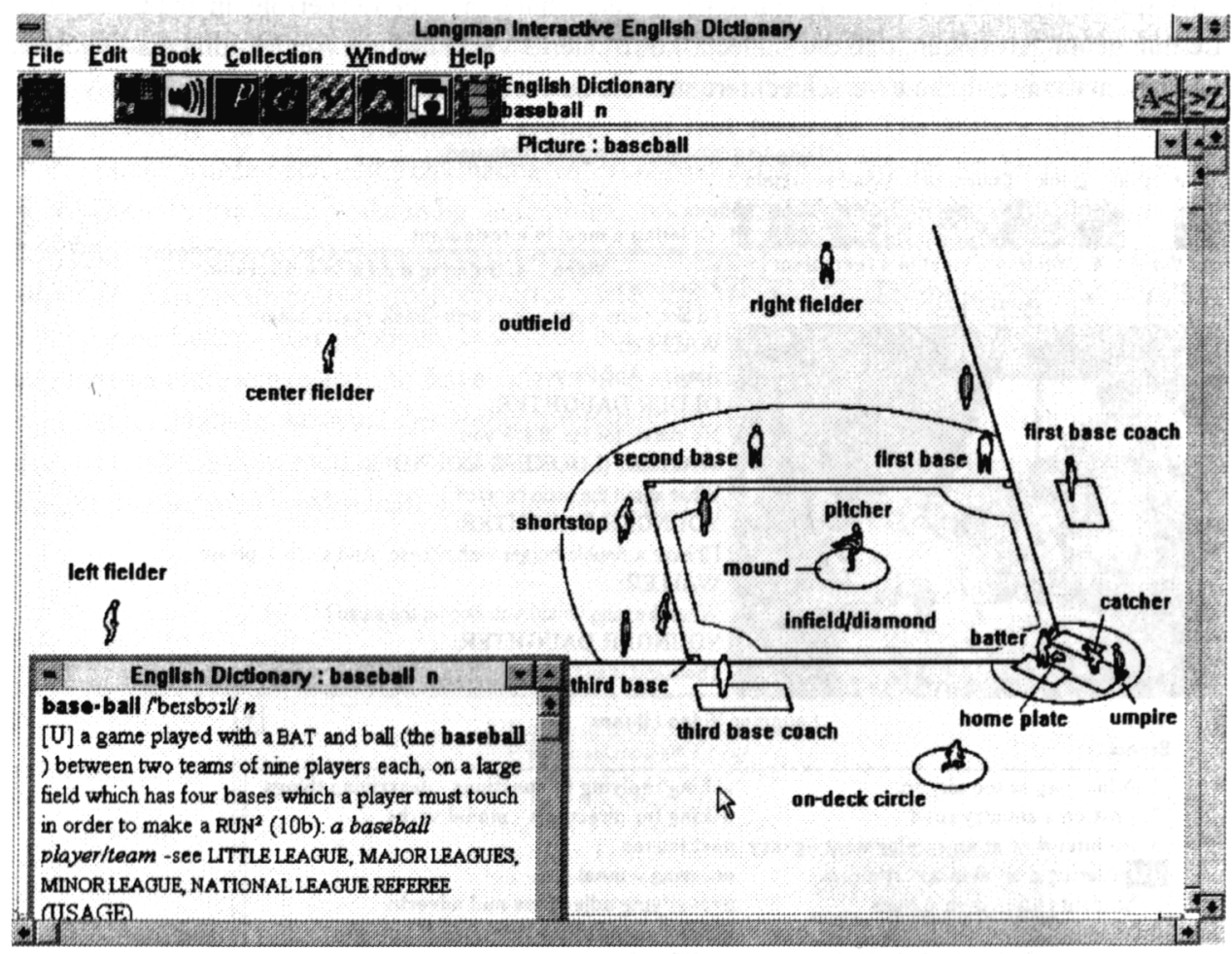

Abb. 2: Bildobjekt zum Eintrag baseball im LIED

Einen ersten Einblick in das vorhandene Innovationspotential gibt das künftig LIED abgekürzte Longman Interactive English Dictionary (LIED 1993), in dessen Mittelpunkt das Longman English Dictionary steht, das mit verschiedenen Zusatzkomponenten zu einem interaktiven Lernerwörterbuch vernetzt ist (vgl. Abschnitt 4.1).

Auch hier werden Bedeutungsparaphrasenangaben um Bilder ergänzt, die zu weiteren zum Wort gehörigen Wortschatzeinheiten führen; so können aus der zum Eintrag baseball (Abb. 2) gehörigen Graphik die Einheiten des Spielfelds und die Benennung der Akteure entnommen werden. In einer „Videothek“ gibt es acht kleine Videosequenzen, zu denen auch der zugehörige Text eingeblendet werden kann (vgl. Abb. 3). Sie sind als Hörverstehensübungen konzipiert, mit denen bestimmte kommunikative Funktionen in prototypischen Situationsabläufen dargestellt werden; in dem in Abbildung 3 gezeigten Beispiel geht es um die Essensbestellung in einem Restaurant. Die bislang spärliche Ausstattung der „Videothek“ zeigt, daß dies erst ein erster Schritt in Richtung Integration von Wörterbuch und Sprachlernumgebung ist; eine Integration, die angesichts der dürftigen Wörterbuchkomponenten von multimedialen Sprachlernsystemen sicher äußerst wünschenswert ist. 
Den multimedialen Aspekt von Hypermedia ausreizen bedeutet nicht, möglichst viele Text-, Ton-, Bild- und Videoobjekte auf einer CD-ROM zusammenzubringen. Wichtig für eine qualitativ hochwertige Anwendung ist vielmehr die Integration der verschiedenen medialen Objekte nach semantisch-funktionalen Prinzipien: „Nur die sinnvolle Kombination, die einen Inhalt unterstützt, hat einen Reiz" ${ }^{6}$ Empirische Untersuchungen zum Lernerfolg in hypermedialen Lernumgebungen zeigen, daß die schlecht koordinierte Verknüpfung von Multimedia-Objekten die Informationsaufnahme verschlechtert statt sie zu verbessern (Weidenmann 1995).

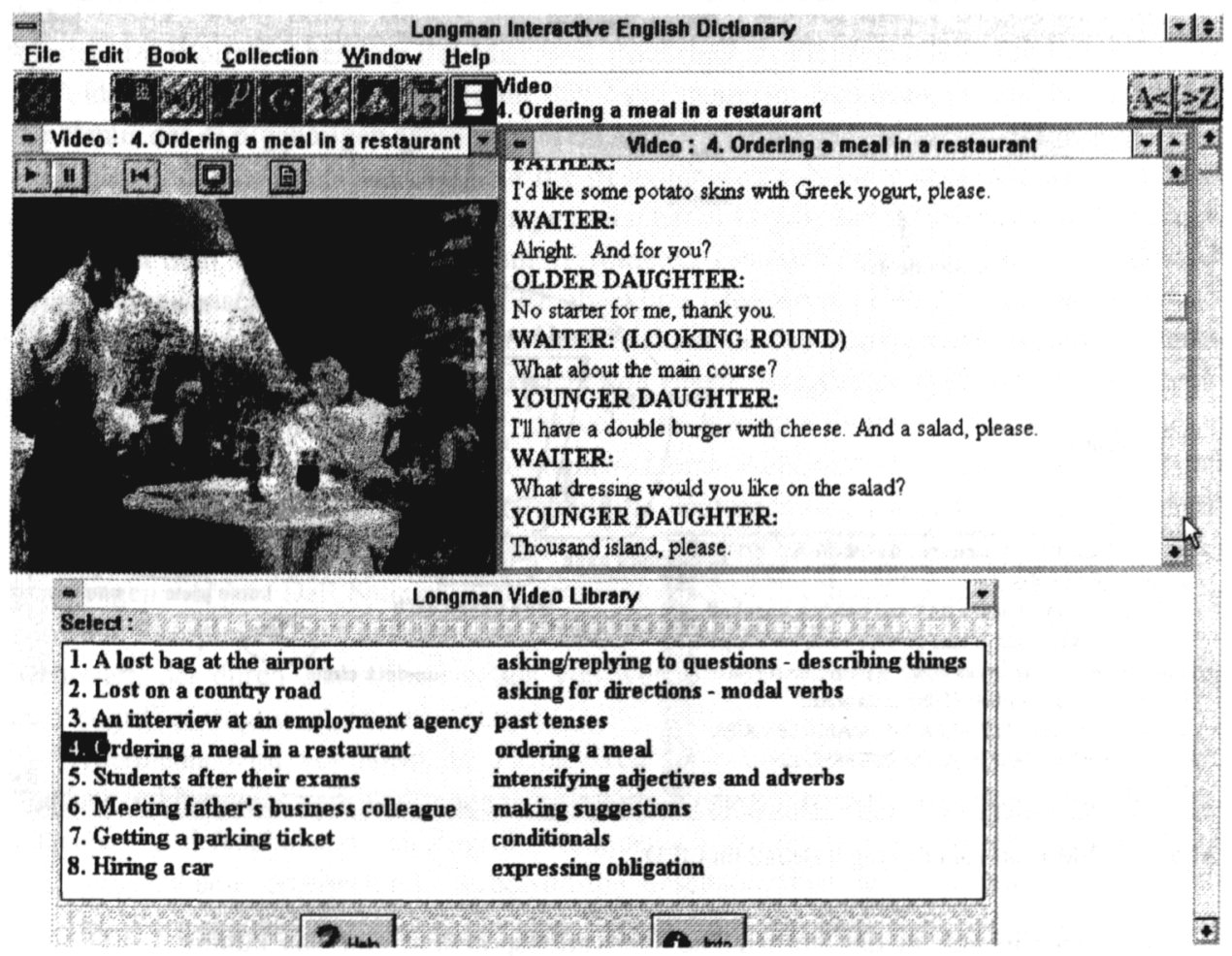

Abb. 3: Hörverstehensübung zum Situationstyp „Essen bestellen“ im LIED

Ein Beispiel für schlechte Integration ist der Langenscheidt's Language Explorer LLE, der sich in der im WWW abrufbaren Produktinformation als Edutainment-Anwendung zu erkennen gibt: „Viel Action mit Spielen und Events zu wechselnden Themen erwarten Euch. (...) Langenscheidts Language Explorer: Surfin' California ist die etwas andere Art, Englisch zu lernen“ (LLE 1997). Allerdings muß das „Edu“ hier mit vielen Fragezeichen versehen werden. Die Metatexte schließen an das vom Fernsehen gewohnte an: Auf verschiedenen sog. „channels“ sind Text, Bild, Musik und Videos zu einem Thema abrufbar; zusätzlich kann in der sog. „Toolbox“ u.a. ein Wörterbuch aufgerufen werden. Die Integration der verschiedenen Komponenten und der multimedialen Objekte läßt allerdings ebenso zu wünschen übrig wie die Qualität der in der Toolbox enthaltenen Informationsangebote. So kann man sich beispielsweise,

6 Vgl. Riehm (1995, 146), zit. nach Schulmeister $(1996,16)$. 
anders als im LIED, zu den Videos keine schriftliche Fassung der gesprochenen Texte anzeigen lassen; man versteht sie oder eben auch nicht. Die vielen bunten Bilder sind zwar mit ergänzenden Texten versehen, beispielsweise die in Abb. 4 gezeigte Text-Bild-Kombination zum Thema „FTP“. Von den im Text benutzten Wörtern führt aber keine Verknüpfung zu den entsprechenden Einträgen des Wörterbuchs. Überhaupt ist das als „Tool“ bezeichnete Wörterbuch kein Werkzeug in dem Sinne, daß man damit alle im Text vorkommenden Wörter nachschlagen kann: $\mathrm{Zu}$ dem in Abbildung 4 gezeigten Text fehlen bspw. die Lemmata abbreviation, advantage, available, browser, dissimilar, recipe, tool und treasure. Die äußere Selektion des Wörterbuchs wurde nach undurchschaubaren Prinzipien getroffen: Weder ist der in den Texten verwendete Wortschatz vollständig aufgeführt, noch enthält das Wörterbuch Einträge wie surf(ing), pool, wave, starlet und producer, die man im Hinblick auf die mit Kalifornien verbundenen Assoziationen nun doch erwartet hätte. Zur inneren Selektion mag als Beispiel der Eintrag zum Lemma star genügen, bestehend aus der grammatischen Angabe „v“, und der Bedeutungsparaphrasenangabe "to have one of the important parts in a movie or play" - eine recht eingeschränkte Auswahl des Bedeutungsspektrums von star. $\mathrm{Da}$ selbst die innovativen Idee, eine offline-Anwendung auf CD-Rom mit online-Diensten des Internet zu verbinden, verschenkt wurde, wird in Abschnitt 4.2 weiter ausgeführt werden.

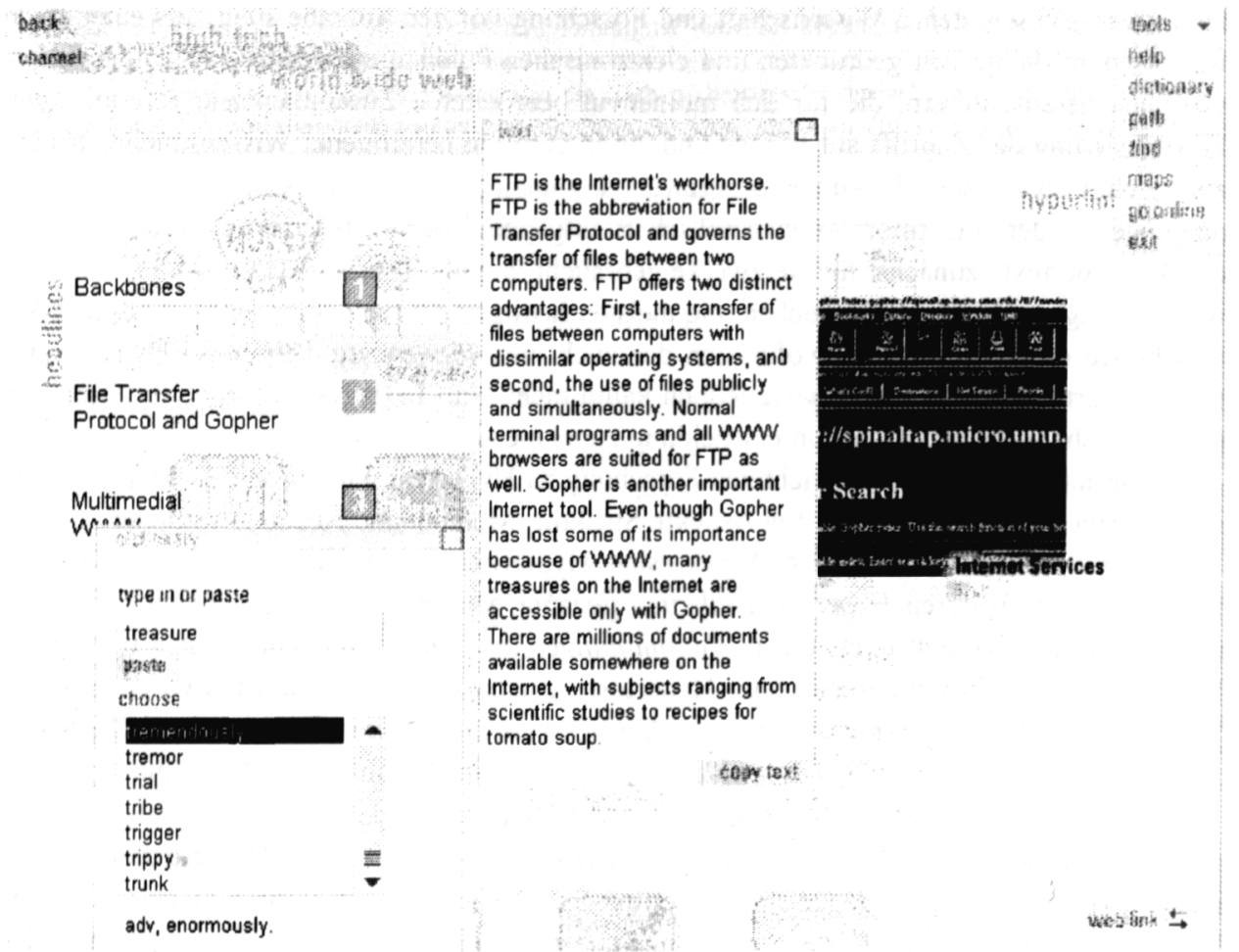

Abb. 4: Multimedia-Kombination zum Thema „high tech“ im LLE (invertiert)

Das Beispiel LLE zeigt ein generelles Problem elektronischer offline-Publikationen, das Schult (1997) folgendermaßen auf den Punkt bringt: „ (...) während man in ein Buch vor dem Kauf 
hineinschauen kann, öffnet sich die CD-Hülle meist erst dann, wenn das Geld schon fort ist. Angesichts der üblen Qualität mancher Produkte scheint das einigen Firmen sogar recht zu sein" (Schult 1997, 115). Rezensionen zu elektronischen Nachschlagewerken sind deshalb wichtig, die Metalexikographie könnte sich hier mit der Erarbeitung von Qualitätskriterien ein neues und wichtiges Aufgabenfeld schaffen.

\section{Hypertext-Wörterbücher: Suchen und Stöbern im lexikalischen Informationsraum}

Als Hypertexte bezeichnet man computerverwaltete Netzwerke von Teiltexten, die über computerisierte Verknüpfungen, sog. Hyperlinks, miteinander verbunden sind. Das Konzept ist seit den 60er Jahren in Informationswissenschaft und Informatik als nichtlineare Form elektronischen Publizierens bekannt, erlangte aber breitere Aufmerksamkeit erst in den letzten Jahren durch das World-Wide-Web (WWW), einen hypertextorientierten Informationsdienst des Internet (s. Abschnitt 5). Mit Hypertext sind Ideen und Informationsutopien verbunden, die jeden faszinieren müssen, der in Wissenschaft und Forschung vor der Aufgabe steht, aus einer rasch wachsenden Menge von gedruckten und elektronischen Publikationen genau diejenigen Informationen herauszufiltern, die für den momentan bearbeiteten Zusammenhang relevant sind. Flexibilisierung des Zugriffs auf Wissen und die Integration heterogener Wissensquellen bis hin zum weltumspannenden Dokumentennetzwerk kommen den Rezeptions- und Produktionsbedingungen in der sog. Informationsgesellschaft entgegen. Historisch gesehen wurde der Ausdruck „Hypertext“ zunächst für Netzwerke gebraucht, die nur Textobjekte enthalten, während, wie im vorigen Abschnitt besprochen, die meisten heutigen Systeme auch Bild-, Ton- und Videoobjekte enthalten können, wofür sich der auch hier verwendete Terminus „Hypermedia“ eingebürgert hat. Da es mittlerweile jedoch kaum mehr Nur-Text-Systeme gibt, verwischt die ursprüngliche terminologische Unterscheidung zunehmend. ${ }^{7}$

In der interdisziplinär ausgerichteten Forschung zu Hypertext gibt es viele Definitionen, die sich auf einen oder mehrere der drei Aspekte Struktur, Medium und Rezeption stützen.

Struktur: Unter strukturellem Aspekt sind Hypertexte beschreibbar als Netzwerke mit Knoten, die im weiteren Hypertext-Einheiten genannt werden. Diese Knoten sind verbunden über (meist) gerichtete Kanten, die als Verknüpfungen (engl. hyperlinks) bezeichnet werden.

Diese Netzwerkstruktur unterscheidet Hypertexte einerseits von zeitlich-linearen Audio- und Videodokumenten; andererseits von gedruckten Dokumenten, deren Struktur durch Teil-Ganzes- und Vorgänger-Nachfolger-Beziehungen zwischen den Dokumententeilen dominiert wird.

Medium: Hypertexte sind an das elektronische Medium gebunden, d.h. Voraussetzung dafür, daß Hypertexte geschrieben und gelesen werden können, ist eine Hypertextsystem genannte Software, die die Menge der Hypertext-Einheiten und die zwischen ihnen angelegten Verknüpfungen verwaltet. Diese Abhängigkeit von einem bestimmten Softwaresystem teilen Hypertexte mit Wörterbuchdatenbanken, die durch ein Datenbanksystem verwaltet werden, das den Zugriff auf die in der Datenbasis gespeicherte Software regelt. In Analogie zur Daten-

7 Vgl. z.B. Kuhlen (1991), Bogaschewsky (1992), Nielsen (1995). 
bankterminologie unterscheidet Kuhlen zwischen dem Hypertextmanagementsystem und den damit verwalteten Hypertextbasen (Kuhlen 1991, 17f.); eine Unterscheidung, die sich in diesem Aufsatz mit den Ausdrücken Wörterbuchsystem vs. Hypertext-Wörterbuch bzw. HypermediaWörterbuch wiederfindet.

Rezeption: Im Hinblick auf die Rezeptionsform werden Hypertexte vom gedruckten Buch auf der einen Seite, von nicht-hypertextualisierten Datenbanken auf der anderen Seite abgegrenzt:

a) Ein gedrucktes Buch erzwingt eine sequentielle Anordnung der Textsegmente, auch wenn diese weder der vom Auto intendierten Leseabfolge noch den von Benutzern individuell eingeschlagenen Lesewegen entspricht. Bei Wörterbüchern haben sich dabei verschiedene „Makrostrukturen“ genannten Anordnungsformen herausgebildet (vgl. Wiegand 1989, Wiegand (im selben Band)), die sich meist an einem Zugriffsalphabeth orientieren. Die Netzwerkstruktur von Hypertexten ermöglicht es stattdessen, verschiedene gleichwertige Zugriffsmöglichkeiten auf die im Hypertext gemachten Angaben anzubieten. Die Verknüpfung der Einheiten des Netzwerks kann intellektuell oder maschinell erfolgen; vom Autor oder vom Leser vorgenommen werden; kann rein assoziativ oder nach semantischen Kriterien erfolgen, z.B. über die Verwendung eines festgelegten Inventars geeigneter Verknüpfungstypen wie „Synonym“, „Antonym“ etc. Der Benutzer folgt den Verknüpfungen, die seinem aktuellen Informationsbedarf und seinen Interessen am besten entspechen.

b) Datenbankorientierte Systeme organisieren den Zugriff auf die Daten über den maschinellen Abgleich zwischen einem Suchmuster und der durchsuchten Datenbasis. Dieses aus dem traditionellen InformationRetrieval stammende sog. „pattern-matching-Paradigma“ wird bei hypertextorientierten Systemen ergänzt um das sog. „explorative Paradigma“, das Durchstöbern einer Datenbasis im Zuge eines nicht genau spezifizierbaren Informationsbedarfs, für das sich die Verben „browsen“, „surfen“ und „navigieren“ eingebürgert haben. ${ }^{8}$ Hypertextsysteme unterstützen die Benutzer dabei, sich über das Verfolgen von Hyperlinks ihren eigenen Weg durch das Netzwerk zu bahnen, dabei Antworten zu einer Frage oder eine klarere Vorstellung von einer sich erst vage abzeichnenden Fragestellungen zu bekommen, oder - wie beim spaßorientierten „surfen“ - sich einfach zu unterhalten, indem ganz im Sinne des Grimmschen Lesebuchgedankens vom Wort zum Spruch, zum Reim und wieder zurück gesprungen wird.

Wörterbücher und Lexika gelten als Vorläufer der Hypertextidee im gedruckten Medium (vgl. z.B. Freisler 1994, Todesco 1995). Dies ist nicht verwunderlich, schließlich weisen Wörterbücher zwei Eigenschaften auf, die sie für Hypertextualisierung geradezu prädestinieren.

1) Lexikographische Textsegmente sind durch explizite und implizite Verweise miteinander verbunden. In Hypertext-Wörterbüchern können diese Verweise durch Verknüpfungen nachgebildet werden, die per Mausklick zum Verweisziel führen.

2) Wörterbücher werden nicht als Ganzes sondern ausschnittsweise rezipiert. Welcher Ausschnitt selektiert wird ist abhängig von der Benutzungssituation und dem dabei verfolgten Zweck. Bei Wörterbuchbenutzungssituationen, in denen bei der Produktion oder Rezeption fremd- oder muttersprachlicher Texte Probleme auftreten, geht es überhaupt nur darum, möglichst rasch und effizient die gesuchte Information zu erschließen. Selbst vom gesamten Wörterbuchartikel werden dabei nur bestimmte Angabeklassen benötigt: die Lemmazeichengestaltangaben bei orthographischen Unsicherheiten, die Bedeutungsparaphrasenangabe bei Verständnisschwierigkeiten etc.. Aber auch die nicht-usuelle Verwendung eines Wörterbuchs als Lesebuch zielt nicht auf vollständige Textrezeption ab, wie die Empfehlung Jakob Grimms zeigt: „Leser jedes Standes und Alters sollen auf den unabsehbaren Strecken der Sprache nach Bienenweise nur in die Kräuter und Blumen sich niederlassen, zu denen ihr Hang sie führt und die ihnen behagen". (Grimm 1854, XII).

Hypertexte unterstützen das selektive Informationslesen und das interessegeleitete Durchstöbern des Wörterbuchs durch entsprechende Such- und Navigationsangebote. Die Suche nach vorgegeben Suchmustern wird sowohl von Hypertext-Systemen als auch von lexikalischen Datenbanken ohne Hypertext-Funktionalität unterstützt. Was ein Hypertext-Wörterbuch einer

8 Vgl. Kuhlen (1991, 25f.). 
Wörterbuchdatenbank voraus hat, ist die Möglichkeit, lexikographische Textsegmente durch Verknüpfungen zu verbinden und damit auch das Durchstöbern von Hypertext-Wörterbüchern im Sinne des o.g. explorativen Paradigma zu unterstützen. Im Anschluß an das Konzept der typisierten Verknüpfungen (Kuhlen 1991, 2.2.5) könnte ein Inventar von Verknüpfungstypen für syntagmatische und paradigmatische Beziehungen, für Wortbildungszusammenhänge und/ oder für Sinnrelationen wie „Antonym“, „Hyperonym“, „Synonym“, für diachrone und synchrone Sprachbeschreibung etc. entwickelt werden, über die zwischen lexikographischen Textsegmente hin- und hergesprungen werden kann.

In Printwörterbüchern sind Informationen, die quer zur primären äußeren Zugriffstruktur liegen, nur dann zu finden, wenn sekundäre Zugriffstrukturen, z.B. verschiedene Register, angeboten werden. Selbst dann muß oft viel geblättert und gesucht werden. Auch kundige Wörterbuchbenutzer dürften Mühe haben, in einem großen gemeinsprachlichen Wörterbuch alle Bewegungsverben, eine verbale Kollokation zu „Erkundung“, den schwäbischen Ausdruck für „Kartoffel“, einen Reim auf „finster“, ein Zitat für die Rede zum Geburtstag der Erbtante oder eine Auswahl idiomatischer Wendungen, mit denen Ärger ausgedrückt werden kann, zu finden. Dem Problem wird in der Welt der Printwörterbücher dadurch abgeholfen, daß verschiedene spezialisierte Sprachnachschlagewerke angeboten werden, z.B. Spezialwörterbücher mit bestimmten Informationstypen (Rechtschreibung, Aussprache, Flexion, Herkunft etc.), syntagmatische und paradigmatische Spezialwörterbücher und auf Varietäten bezogene Wörterbücher (Sprachstadien, Dialekte, Fachsprachen, Gruppensprachen etc.). ${ }^{9}$ Wären lexikographische Angaben verschiedenster Art durch typisierte Verknüpfungen miteinander verbunden, könnten die bislang in getrennten Büchern beschriebene Aspekte sprachlichen Wissens in einem HypertextWörterbuch vereint werden. Dieses könnte die gezielte Suche nach bestimmten Angabenklassen genauso unterstützen wie das Durchstöbern des lexikalischen Informationsraums durch Verfolgen der typisierten Verknüpfungen. In den meisten Fällen würde wohl eine kombinierte Strategie zum Tragen kommen, die zunächst über ein Suchmuster eine Vorauswahl trifft und von dieser ausgehend durch das Verfolgen von Hyperlinks weitere Erkundungen unternimmt.

Ein erster Schritt in diese Richtung sind Wörterbuchsysteme wie die PC-Bibliothek oder das Bee-Book-System. Beide Systeme ermöglichen es, mehrere Nachschlagewerke gleichzeitig zu durchsuchen. Vor allem die PC-Bibliothek verfügt über sehr flexible Suchoptionen und ermöglicht dadurch im Vergleich zum gedruckten Medium neuartige Zugriffsformen auf das lexikalische Wissen: Es kann wahlweise nur nach Lemmata gesucht oder eine Volltextsuche über den gesamten Wörterbuchtext gestartet werden. Lobenswerte Extras, die beispielsweise im Bee-Book-System fehlen, sind einerseits sog. „Jokerzeichen“, im Computerjargon „wildcards“ genannt, die in Suchmustern für beliebige Zeichen oder Zeichenfolgen eingesetzt werden können, andererseits die Möglichkeit, mehrere Suchmuster über boolsche Operatoren miteinander zu verknüpfen und schließlich die schreibtolerante Suche, die ausgehend von Suchmustern wie „Koriphäe“ oder „Korifäe“ zu Koryphäe findet, und damit dem Problem abhilft, daß man ein Wort deshalb nicht nachschlagen kann, weil man dessen korrekte Schreibweise nicht kennt. Beide Systeme unterstützen über diese Suchfunktionen vornehmlich den datenbankorientierten Zugriff nach dem ,pattern-matching-Paradigma“, während die Möglichkeiten zur Informationssuche nach dem explorative Paradigma noch viele Wünsche offen lassen. Verknüpfungen zwischen Wörterbuchartikeln nach inhaltlichen oder pragmatisch-funktionalen Kriterien werden

9 Vgl. die Einteilung in Hausmann/Wiegand (1989), Hausmann/Reichmann et al. (1990). 
selten angeboten, statt dessen werden Verknüpfungen nach formalen Kriterien automatisch generiert.

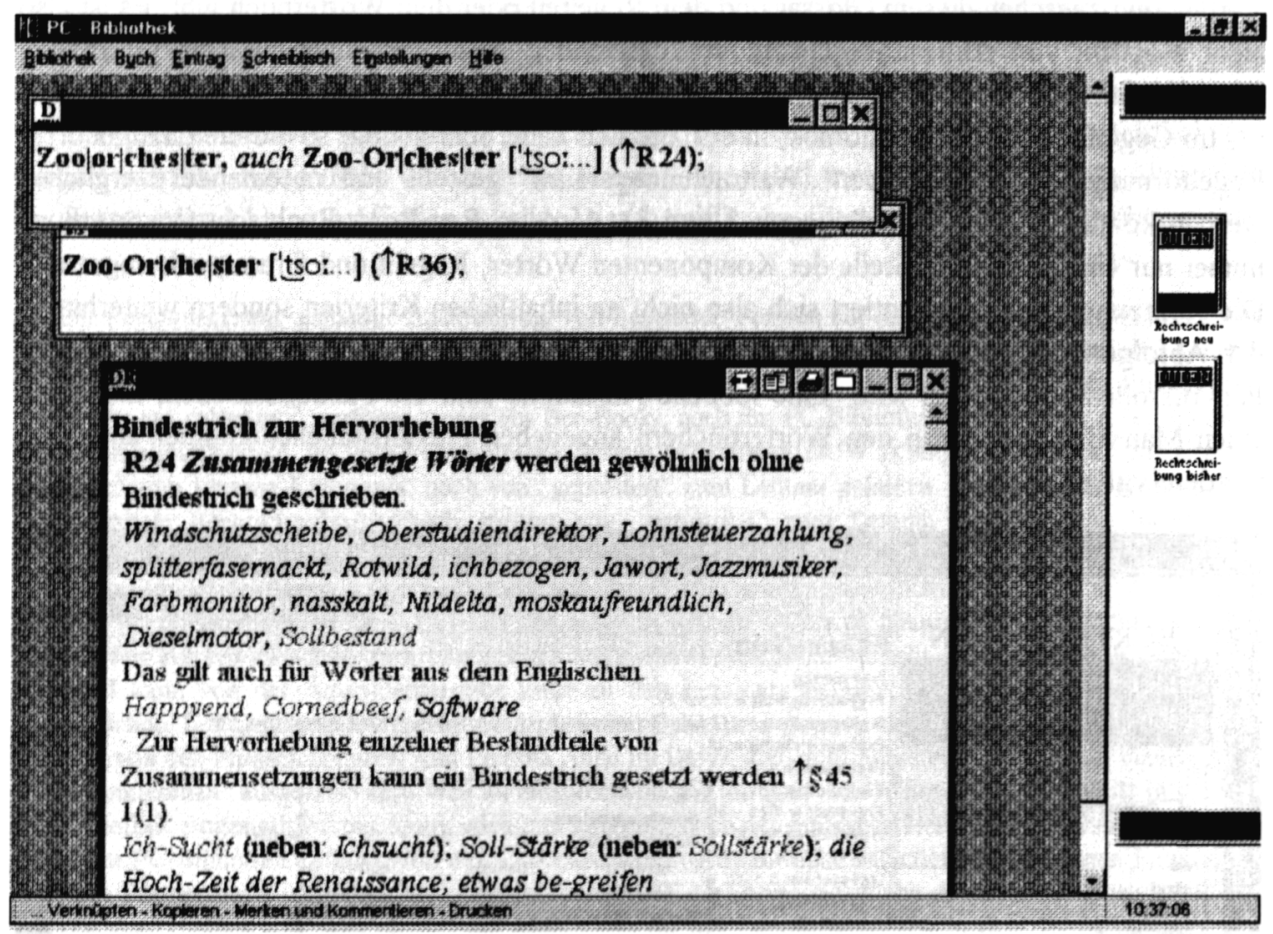

Abb. 5: Zooorchester in alter und neuer Schreibung in der PC-Bibliothek

Ein Mausklick auf eine beliebige Zeichenkette zwischen zwei Leerzeichen startet in der PC-Bibliothek beispielsweise eine Stichwortsuche, in der das aktivierte Textsegment als Suchmuster fungiert. Die Fundstellen werden dann in einem Auswahlfenster angezeigt. Dieses Verknüpfungsprinzip liegt selbst expliziten Verweisen zugrunde, die wie im gedruckten Wörterbuch mit einem Verweispfeil gekennzeichnet sind. Die beim Lemma wich angeführte Verweisangabe „weichen(2)" führt also nicht etwa direkt zum Lemma weichen(2), sondern zu einer Auswahlliste, in der die Homonyme weichen(1) und weichen(2) aufgeführt sind.

Ein Schritt in die richtige Richtung ist das ebenfalls in die PC-Bibliothek einstellbare Rechtschreibwörterbuch (DUDEN), in dem bisherige und neue Schreibungen systematisch miteinander verknüpft sind. Beispielsweise kann man von dem in Abbildung 5 gezeigten Artikel ZooOrchester aus dem „alten“ Rechtschreib-Duden ausgehend zum Artikel Zooorchester des „reformierten" Rechtschreib-Dudens und von dort aus über eine durch den Verweispfeil angezeigte Verknüpfung zu derjenigen Regel springen, durch die die alte und neue Bindestrichschreibung „Zoo-Orchester“" weiter zugelassen wird.

Eine derartige Verbindung zwischen Regel und Wörterbuch bietet auch das in das BeeBook-System einstellbare Bertelsmann Rechtschreibwörterbuch BeeBook (nR) (1996) in dem man z.B. von dem in Abbildung 6 gezeigten Eintrag zu Freitagabend aus zu den Paragraphen 37 (1) und 56 (3) im amtlichen Regelwerk springen kann. Leider werden derartige Sprung- 
möglichkeiten nur selten und wenig systematisch angeboten. So ist das Vorhandensein eines Glossars zu grammatischen Termini zwar begrüßenswert; es ist aber schade, daß es keinerlei Verbindung zwischen diesem Glossar und dem Regelteil oder dem Wörterbuch gibt. Es ist also nicht möglich, von den im Regelwerk vorkommenden Ausdrücken wie „Partikel“, „Präfix“ oder „Diphthong“ ausgehend zu deren Erläuterungen im grammatischen Glossar zu springen.

Im Gegensatz zur PC-Bibliothek, in der alte und neue Schreibung, sowie die dazugehörige Regelformulierung in denselben „Wahrnehmungsraum“ gestellt und miteinander verglichen werden können (vgl. die Abbildungen 5 und 8), wird im Bee-Book-Rechtschreibwörterbuch immer nur eine bestimmte Stelle der Komponenten Wörter, Regeln und Grammatik angezeigt. Die Kontextualisierung orientiert sich also nicht an inhaltlichen Kriterien sondern weiterhin an der Abfolge der Textsegmente in den entsprechenden Druckwerken, die als „elektronische Papyrusrollen“ organisiert sind. Eine löbliche Ausnahme sind die Flexionstabellen, die durch einen Mausklick auf die in den Wörterbüchern angegeben Flexionstabellennummer angezeigt werden (vgl. Abb. 6).

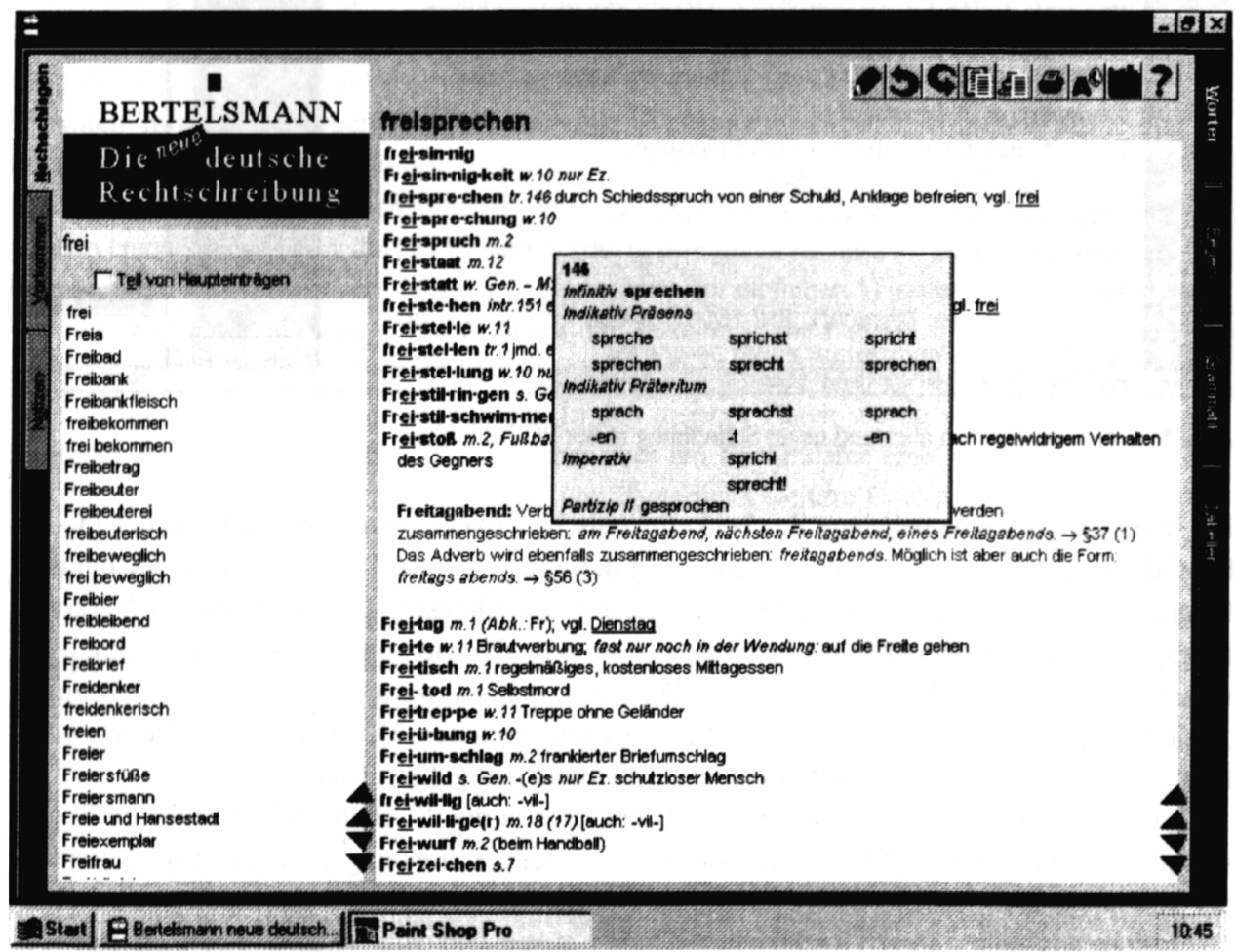

Abb. 6: Freitagabend im Bee-Book-System

Bislang dominieren in beiden Systemen die automatisch generierten Verknüpfungen, bei denen das Potential der oben skizzierten Hypertextidee noch wenig genutzt wird. Trotzdem entsteht dadurch ein Mehrwert gegenüber dem gedruckten Buch, der vor allem nicht-muttersprachlichen Wörterbuchbenutzern entgegenkommen dürfte. Denn diese verstehen häufig Wörter, die zur Bedeutungserläuterung eines unbekannten Wortes genutzt werden, ebenso wenig wie das 
gesuchte Wort selbst. In diesem Fall ist es von Vorteil, wenn durch einen einfachen Mausklick nach dem zugehörigen Wörterbuchartikel gesucht werden kann. Auch in solchen Situationen ist das PC-Bibliothek-System, das mehrere Wörterbuchartikel in verschiedenen „Fenstern“ gleichzeitig auf dem Bildschirm anzeigen kann, m.E. dem Bee-Book-System überlegen, bei dem immer nur ein einzelner Ausschnitt aus einem Nachschlagewerk angezeigt wird.

Der Gebrauchswert automatisierter Verknüpfungen könnte jedoch noch beträchtlich gesteigert werden, wenn mehr vorhandenes linguistisches und metalexikographisches Wissen in die Systementwicklung einfließen würde. Dies betrifft vor allem die folgenden Problembereiche:

1) Die Lemmatisierung genannte Rückführung von flektierten Formen auf ihre Grundform ist Voraussetzung dafür, daß die automatisch generierten Verknüpfungen in allen Fällen korrekt hergestellt werden können. Obwohl das Problem der Lemmatisierung in der Computerlinguistik mit Ausnahme einiger Problemfalle bereits gut gelöst ist ${ }^{10}$, verfügen weder das Bee-Book-, noch das PC-Bibliothek-System über eine Lemmatisierungskomponente. Im vom PC-Bibliothek-System verwalteten DUW gelangt man weder von „Erzeugnisse“ zum Lemma Erzeugnis; noch von „gepolstert“ zum Lemma polstern. Das vom Bee-Book-System verwaltete Rechtschreibwörterbuch springt von „geworden“ zum Lemma Gewühl, die Suche nach "gelieferte“ führt zum Stichwort gelieren. Für stark flektierende Sprachen wie das Deutsche vermindert dieser Mangel den Nutzwert der automatischen Verknüpfungsfunktion beträchtlich.

2) Mehrwortlexeme können nicht als Einheiten aktiviert werden, da die automatisierte Verknüpfung sich immer nur auf ein zwischen zwei Leerzeichen stehendes Textsegment bezieht. Vom Lemma Kartoffel im TWB kann von der Äquivalentangabe zwar zu den Lemmata pomme, de und terre im französischdeutschen Teil gesprungen werden, nicht aber zum Sublemma pomme de terre direkt. Ebenso führt der Weg von den Phrasemangaben zum Lemma Auge im DUW, z.B. vom Sublemma „mit einem blauen Auge davonkommen" ausgehend kein Weg zu entsprechenden Einträgen des Duden-11, der ebenfalls in die PCBibliothek eingestellt werden kann, auch hier kann nur nach den einzelnen Bestandteilen gesucht werden.

3) In der PC-Bibliothek differenziert das Verknüpfungsprogramm nicht zwischen verschiedenen Funktionen eines Textsegments im Wörterbuchartikel. Ob im DUW die Polysemieangabe „, ", ob der Buchstabe „B“ als Teil des Lemmas z.B. oder ob „b.“ als Abkürzung für das Lemma bewegen in den Beispielangaben des Artikels aktiviert wird; stets startet dieselbe Suche nach dem Suchmuster „, langen Fundliste mit allen „b“ enthaltenden Lemmata von A.B. bis z.B.V. Hier schneidet das Bee-BookSystem besser ab. Abkürzungen für grammatische Angaben oder Fachbereichsangaben werden als solche erkannt; ihre Vollform läßt sich per Mausklick anzeigen, im TWF sogar in beiden Sprachen.

Allerdings muß man sich fragen, was Abkürzungen und Hilfssymbole zur Textkondensation, z.B. die Abkürzung des Lemmazeichens durch das Initial in den Beispielangaben des DUW, überhaupt noch in elektronischen Wörterbüchern zu suchen haben. Ein enormer Vorteil der Computerisierung von Wörterbüchern besteht ja - ganz unabhängig von der Hypertextidee genau darin, daß nicht mehr mit teurem Druckraum gespart werden muß (vgl. Breidt (im selben Band); Lemberg/Petzold et al. (im selben Band)). Die Tatsache, daß 20 Bände Oxford English Dictionary (OED) auf eine CD-Rom gebrannt werden können (s.u.), zeigt, daß für die wenig speicherintensiven Textobjekte genügend Platz ist - es darf also ruhig eine Angabe mehr sein. Das aus der Print-Lexikographie bekannte Dilemma, daß das Ziel, eine möglichst hohe Anzahl von Daten auf eine Druckeinheit zu bringen, ein hohes Ausmaß an Textverdichtung erfordert, daß diese Verdichtung aber die Verständlichkeit der lexikographischen Angaben enorm erschwert (vgl. Wiegand 1987, 37), besteht im neuen Medium nicht mehr.

Das Noch-Vorhandensein entsprechender Textverdichtungsmittel deutet darauf hin, daß die elektronischen Wörterbücher der PC-Bibliothek und des Bee-Book-Systems durch eine relativ

10 Z.B. in dem in Breidt (im selben Band) diskutierten System COMPASS; einen Überblick über aktuelle Systeme zur morphologischen Analyse und zur Lemmatisierung im Deutschen gibt Hausser (1996). 
oberflächliche Bearbeitung der gedruckten Wörterbücher entstanden sind. Der maschinenlesbare Ausgangstext wurde nur sehr grobkörnig segmentiert in die Wörterbuchartikel, die Grundbausteine des Wörterteils, in denen dann weiter zwischen Lemmazeichengestaltangabe und Restartikel unterschieden wurde. Im DUW wurden zusätzlich die „Nischenartikel“"11 in eigenständige Artikel umgewandelt und die entsprechenden Lemmata in die Stichwortliste aufgenommen. Dabei sind sowohl die metalexikographischen Voraussetzungen als auch die computertechnischen Möglichkeiten für eine feinkörnigere Analyse der Wörterbuchartikel vorhanden. Die metalexikographischen Grundlagen für die Beschreibung von Wörterbuchstrukturen hat H.E. Wiegand in seiner Theorie lexikographischer Texte gelegt. ${ }^{12}$ Dieser theoretische und terminologische Rahmen kann genutzt werden, um mit einem als Wörterbuchparser bezeichneten Softwaresystem sog. Wörterbuchdatenbanken zu erzeugen, in denen die hierarchische Struktur der Wörterbuchartikel so expliziert ist, daß auf die einzelnen lexikographischen Textsegmente gezielt zugegriffen werden kann. ${ }^{13}$ Diese können dann nach Bedarf nacheditiert werden, indem z.B. Abkürzungen und andere Textkondensationsmittel aufgelöst werden und indem die überarbeiteten Textsegmente nach verschiedenen Gesichtspunkten miteinander verknüpft, um sinnvolle multimediale Objekte ergänzt (vgl. Abschnitt 2) und damit in „richtige“ Hypertext-Einheiten umgewandelt werden. Wie diese Überarbeitung zumindest teilautomatisiert werden kann, beschreiben Thielen et al. und Breidt (im selben Band) am Beispiel der COMPASS-Wörterbuchdatenbank. Überlegungen zur Hypertextualisierung gedruckter Wörterbuchtexte auf der Basis der in Wiegand (1996) beschriebenen wörterbuchinternen Mediostrukturen finden sich in Kammerer (1997).

Ein auf der Basis einer geparsten Wörterbuchdatenbank erstelltes elektronisches Wörterbuch würde schon im Bereich der Suchoptionen völlig neue Möglichkeiten eröffnen. Wer im DUW nach dem regionalen Bezeichnungen für „Kartoffel“ fahndet, indem er eine Volltextsuche mit „Kartoffel“" startet, findet bislang zwar die Einträge Grundbirne, Erdapfel, Erdbirne, zusätzlich aber auch Auge, denn Kartoffeln haben Augen, und Himmel, denn in einer als „Himmel und Erde“ bezeichneten Speise sind u.a. Kartoffeln enthalten (vgl. Abb. 8). Die Volltextsuche nach „,bewegen“, mit der Hoffnung gestartet, möglichst viele deutsche Bewegungsverben zu finden, zeigt eine lange Liste in der u.a. auch Anmut, affenartig, Gefühl, und geschmeidig auftauchen, weil „bewegen“ in deren Bedeutungsparaphrasenangabe enthalten ist; im Artikel zu Bein findet sich das Suchwort in der Bedeutungserläuterung der Phrasemangabe „etw. geht in die Beine“, Lemmata wie Genick und hurra verdanken ihr Auftauchen in der Liste schließlich dem Vorhandensein des Suchworts in der etymologischen Angabe; die Volltextsuche in einem ungeparsten Wörterbuch liefert also wenig präzise Ergebnisse.

Eine gezielte Suche in den Bedeutungsparaphrasenangaben erlaubt beispielsweise das elektronische Oxford English Dictionary (OED) das nach dem in Raymond/Tompa (1988) beschriebenen Konversionsverfahren in ein Hypertext-Wörterbuch überführt wurde. Das OED läßt erahnen, welches Potential in einem feinkörniger segmentierten und nach inhaltlichen und funktionalen Gesichtspunkten relationierten Wörterbuch steckt. ${ }^{14}$ Das OED ist ein großes gemeinsprachliches Wörterbuch, das in mehr als 600000 Einträgen den englischen Wortschatz

$11 \mathrm{Zu}$ Nest- und nischenalphabetischen Anordungsformen vgl. Wiegand (1989).

12 Vgl. u.a. Wiegand $(1983,1987,1989,1991,1996$ u. 1997).

13 Vgl. Bläsi/Koch (1992), Hauser/Storrer (1994, 1996), Storrer (1996).

14 Das OED lag mir nicht vor; ich stütze mich deshalb auf die in Cölfen (o.J.) gegebene Funktionsbeschreibung. 
vom 12. Jahrhundert bis in die Gegenwart beschreibt und 2,4 Millionen Belege aus allen Sprachstadien und verschiedenen Textsorten enthält. Obwohl auf multimediale Zusätze gänzlich verzichtet wird, eröffnet das elektronische OED neben der gängigen Stichwort- und Vollformensuche Zugriffsmöglichkeiten, die weit über diejenigen herkömmlicher elektronischer Wörterbücher hinausgehen: So kann z.B. gezielt nach bestimmten Lemmatypen wie Redewendungen, Prä- und Suffixen gesucht werden; die Suche kann sich auf ein Sprachstadium oder sogar ein konkretes Datum beziehen und Suchanfragen können nicht nur mit dem lateinischen, sondern auch mit dem phonetischen Alphabet formuliert werden. Zusätzlich kann sich der Benutzer entscheiden, ob er nur die Bedeutungserläuterung sehen möchte, oder auch Belege und etymologische Angaben.

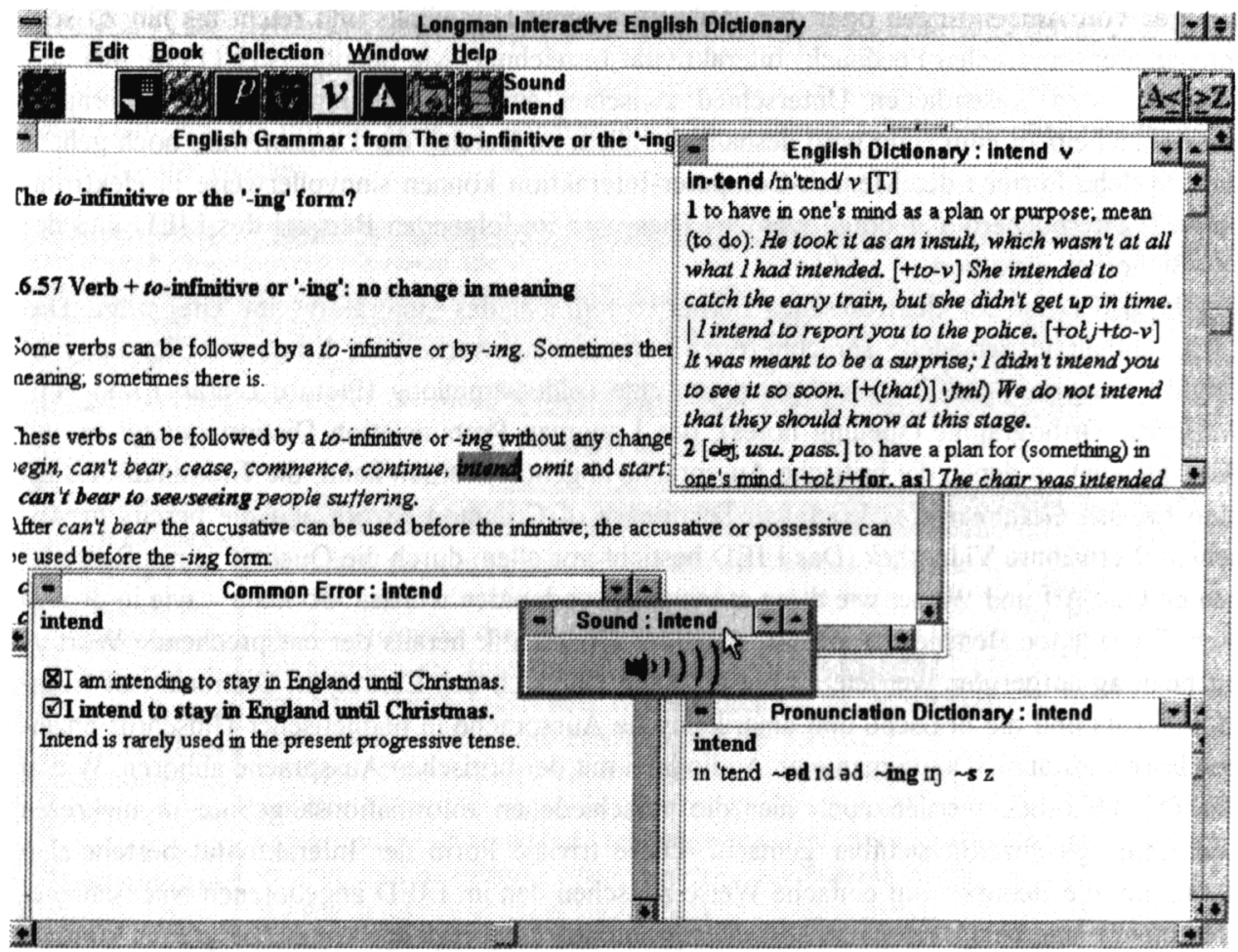

Abb. 7: Informationen zu intend im LIED

Sorgfältig segmentierte, nach funktional-inhaltlichen Gesichtspunkten relationierte und mit entsprechenden Navigationswerkzeugen ausgestattete Hypertextwörterbücher sollten auch für das Deutsche entwickelt werden. Wären diese zudem noch mit intelligenten Nachschlagefunktionen versehen, wie das in Breidt (im selben Band) beschriebene COMPASS-System, entstünden flexibel nutzbare elektronische Wörterbücher, an denen sicherlich auch Jakob Grimm seine Freude hätte. 


\section{Wörterbücher in Aktion}

\subsection{Interaktive Wörterbücher}

Der aus den Sozialwissenschaften stammende Begriff „Interaktion“ wurde in den 80er-Jahren auch auf die Beziehung zwischen Mensch und Computer übertragen Als ,interaktiv“ bezeichnet man in diesem Zusammenhang Software, die auf Eingaben eines Anwenders in vorprogrammierter Weise reagiert (vgl. Haak 1995). Die Formen dieser „Interaktion“ reichen von einfachen Operationen wie dem Betätigen eines Sprungknopfes oder dem Treffen einer Auswahl aus einer Liste über die Adaption und Individualisierung der Anwendung durch das Hinzufügen von Anmerkungen oder dem Anlegen eigener Hyperlinks und reicht bis hin zu sog. intelligentem tutoriellen Feedback. Interaktivität bezeichnet, wie Schulmeister $(1996,40)$ herausstellt, „den wesentlichen Unterschied zwischen einem computerunterstützten Lernprogramm und einem Film“ und wird deshalb vor allem im Bereich der Lernsoftware hoch gehandelt. Welche Formen der Mensch-Computer-Interaktion können sinnvollerweise in elektronischen Wörterbüchern angeboten werden? Dies wird im folgenden Beispiel des LIED und der PC-Bibliothek diskutiert.

Das LIED ist ein elektronisches Lernerwörterbuch, das „interaktiv“ im Titel trägt. Das LIED enthält verschiedene sprachbezogene Nachschlagewerke: das Longman Dictionary of English Language and Culture ergänzt um eine Bildersammlung (Picture Library) und Hinweise zur Orthographie (spelling notes), das Longman Pronunciation Dictionary ergänzt um Audiodateien, in denen die britische Aussprache abgehört werden kann, die Grammatik Longman English Grammar, das Longman Dictionary of Common Errors und die bereits in Abschnitt 2 erwähnte Videothek. Das LIED besticht vor allem durch die Qualität seiner Ressourcen und die Art und Weise, wie diese miteinander verbunden wurden. So kann - wie in dem in Abb. 7 gezeigten Beispiel zu intend - aus der Grammatik heraus der entsprechende Wörterbucheintrag aufgerufen werden; zu diesem kann man sich den Eintrag im Dictionary of Common Errors und die britische und amerikanische Aussprache in phonetischer Umschrift anzeigen lassen, zusätzlich kann man eine Audiodatei mit der britischen Aussprache abhören. Wie in der PC-Bibliothek werden auch hier die verschiedenen Informationsangebote in mehreren „Fenstern“ gleichzeitig sichtbar gemacht. Diese triviale Form der Interaktivität besteht also darin, daß die Benutzer auf einfache Weise zwischen den im LIED angebotenen Nachschlagewerken hin- und herspringen und sich interessante Informationsangebote herausgreifen können. In diesem Sinne sind aber alle besprochene Wörterbücher „interaktiv“.

Interaktivität kann aber noch mehr sein, kann die aktive Auseinandersetzung der Benutzer mit den im Wörterbuch enthaltenen Informationsangeboten fördern und ihnen die Möglichkeit geben, das System an die individuellen Interessen, Wissensvoraussetzungen und Bedürfnisse anzupassen. Ein Interaktionsangebot in diesem Sinne stellt das LIED durch die Funktion "collection" bereit, durch die die Benutzer Text-, Ton-, Bild- und Videoobjekte nach selbst gewählten Gesichtspunkten zusammenstellen und später wieder abrufen können. Ein Lehrer kann also beispielsweise eine Hörverstehensübung aus der Videothek herausgreifen und sich dazu von den Schülern Sammlungen zum unbekannten Wortschatz und Angaben zu grammatischen Schwierigkeiten anlegen lassen. Allerdings kann im LIED nur gesammelt werden; An- 
merkungen zu diesen Sammlungen, eigene Wörterbucheinträge, die Verbindung von Wörterbucheinträgen mit selbst gefundenen Belegstellen u.a. können nicht erstellt werden.

Wesentlich weiter geht hier die PC-Bibliothek: Jeder Eintrag kann mit einem Kommentar versehen und einem oder mehren selbstgewählten Schlagwörtern zugeordnet werden. Der dabei entstehende elektronische Schlagwortkatalog bietet natürlich wesentlich komfortablere $\mathrm{Zu}$ griffs- und Verwaltungsmöglichkeiten als herkömmliche Zettelkästen. Weiterhin können Benutzer eigene Verknüpfungen zwischen den Wörterbuchartikeln anlegen, die sich über automatisch eingefügte Verknüpfungsanzeiger aktivieren lassen. Abbildung 8 zeigt den Eintrag zu Kartoffel, der mit den entsprechenden regionalsprachlichen Bezeichnungen verknüpft ist. Auch wenn die Bedienung der Karteikasten- und der Verknüpfungsfunktionen noch etwas benutzerfreundlicher gestaltet werden könnte, unterstützt die PC-Bibliothek den kreativen und interaktiven Umgang mit den Angaben der Nachschlagewerke. Die Möglichkeit, elektronische Schlagwortkataloge oder nach selbstgewählten Prinzipien verküpfte Hypertexte aufzubauen kann nicht nur für private Zwecke, sondern auch im Rahmen schulischer oder universitärer Lehre genutzt werden.

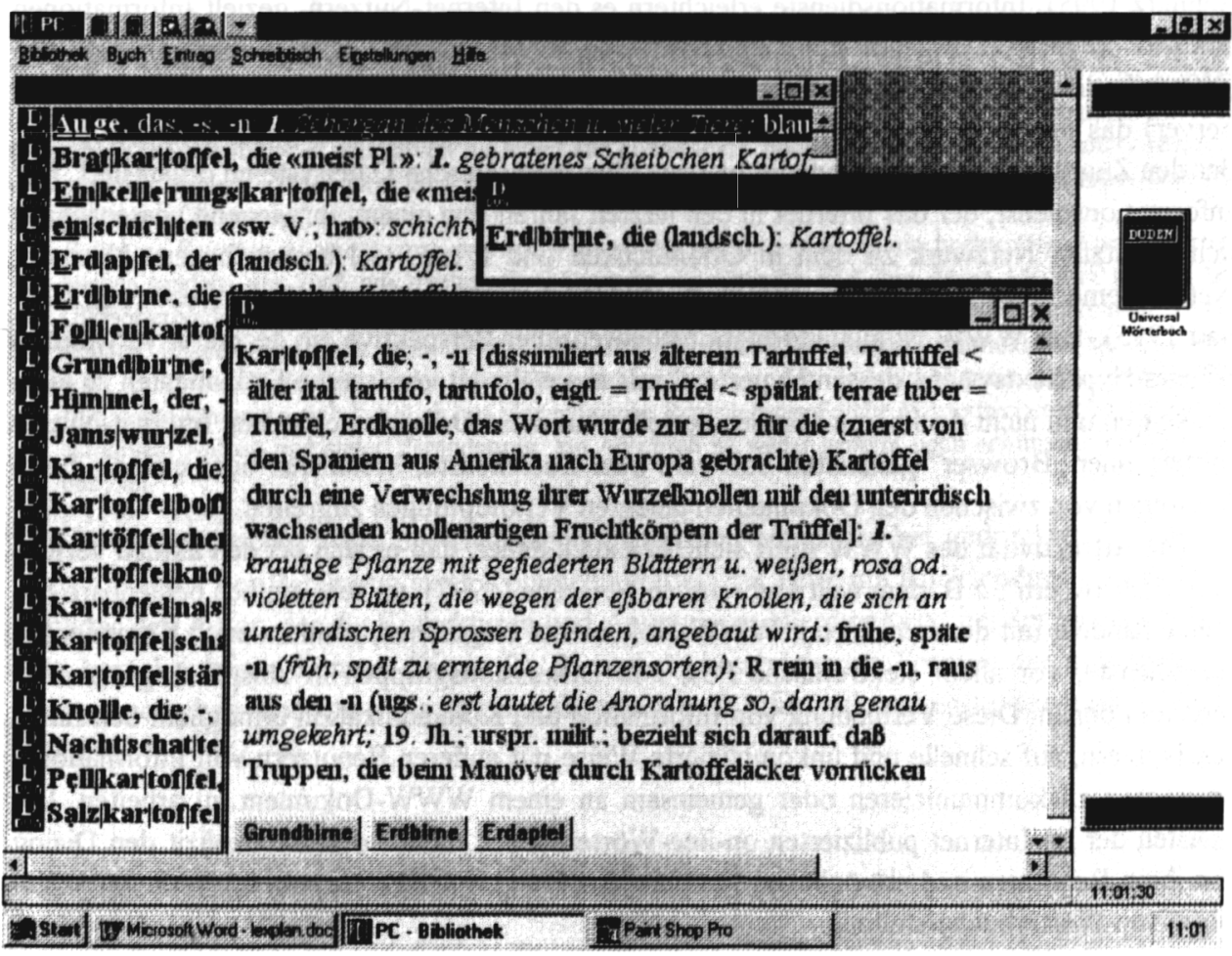

Abb. 8: Benutzerdefinierte Verknüpfungen zu Kartoffel in der PC-Bibliothek 


\subsection{Kollaborative Wörterbucherstellung im WWW}

Neuere Entwicklungen in der Computertechnik haben nicht nur die „Interaktion“ zwischen Mensch und Maschine vereinfacht und den Aktionsspielraum der Computerbenutzer erheblich erweitert. In Computernetzwerken wie dem weltumspannenden Internet entstehen auch neue Formen der computerunterstützen Interaktion zwischen den durch das Netzwerk verbundenen Benutzern (vgl. Herring 1996). Das Internet ist ein dezentraler Verbund von Rechnernetzen, über den mehrere Millionen Rechner aus der ganzen Welt Daten austauschen und miteinander kommunizieren können. ${ }^{15}$ Die an das Internet angeschlossenen Rechner können, entsprechende Software vorausgesetzt, verschiedene sog. Dienste nutzen, die sich unter funktionalem Gesichtspunkt in Kommunikations- und Informationsdienste unterteilen lassen. Zu den Kommunikationsdiensten zählt die elektronische Post (engl. „e-mail“) die elektronischen Nachrichtenbretter (engl. „Newsgroups“), in denen Neuigkeiten und Meinungen zur allgemeinen Diskussion gestellt werden können, sowie die Online-Diskussionsgruppen (engl. „Chat"), in denen verschiedene Teilnehmer über Tastatureingaben miteinander „schwatzen“ können (vgl. Lenke/ Schmitz 1995). Informationsdienste erleichtern es den Internet-Nutzern, gezielt Informationen aus den im Internet angebotenen Datenbeständen herauszufiltern; dazu gehören Dienste wie „Whois“ oder „Netfind“ mit Metainformationen zum Internet, „WAIS“ (Wide Area Information Server) das die Volltextsuche in weltweit verteilten Datenbanken ermöglicht oder „Gopher“, der den Zugriff auf Datenbestände im Internet über hierarchische Dateisysteme organisiert. Der Informationsdienst, der das Internet in den letzten Jahren von einem vorwiegend wissenschaftlich genutzten Netzwerk zu dem in Öffentlichkeit und Presse viel besprochenen „Netz der Netze" gemacht hat, ist das im weiteren als WWW abgekürzte „World Wide Web“ (vgl. Cailliau 1995). Das WWW ist aus informationstheoretischer Perspektive ein dezentral verwaltetes offenes Hypertextsystem, dessen Hypertextbasis aus weltweit verstreuten Dokumenten zu allen denkbaren und nicht denkbaren Themen besteht. Auf diese Dokumente können Internet-Nutzer mittels einer „Browser“ genannten Software über Suchdienste, Metalisten oder einfach durch Verfolgen von zwischen den Dokumenten gelegten Verknüpfungen zugreifen.

Die Attraktivität des WWW rührt sicherlich auch daher, daß es sich bei den aktuell verfügbaren „Browsern“, z.B. dem weit verbreiteten Netscape (1997) um sehr einfach bedienbare Systeme handelt, mit denen nicht nur WWW-Dokumente eingesehen, sondern auch Kommunikationsdienste, vor allem elektronische Post und Diskussionsgruppen in Anspruch genommen werden können. Diese Verbindung von Information und Kommunikation ermöglicht den Internet-Nutzern, auf schnelle und unkomplizierte Weise mit anderen Benutzern von Informationsangeboten zu kommunizieren oder gemeinsam an einem WWW-Dokument zu arbeiten. Die meisten der im Internet publizierten on-line-Wörterbücher suchen deshalb explízit den Dialog mit ihren Benutzern und ermöglichen verschiedene Formen der Partizipation bis hin zur kollaborativen Wörterbucherstellung:

1) Fehlerbehebung: Häufig werden die Benutzer von WWW-Wörterbüchern explizit zur Kommentierung und zur Anzeige von inhaltlichen oder orthographischen Fehlern aufgefordert. Ein typischer Aufruf dieser Art entstammt dem Wörterbuch der Rechtssprache der World Wide Legal Information Association WWLIA (1995): „We welcome your comments, words of wisdom or encouragement, constructive criticism, advice,

15 Gut verständliche Einführungen ins Internet sind Kretschmer (1996) und Cölfen/Cölfen et al. (1997). 
suggestions or just your kindness in pointing out a grammar or spelling mistake which you have found. Click here to send an e-mail to the Webmaster of the World Wide Lega Information Association.".

2) Schließung von Lemmalücken: In anderen WWW-Wörterbüchern bitten die Autoren die Benutzer, sie über fehlende Eintrăge zu benachrichtigen bzw. sich durch eigene Vorschläge an der Schließung von Lemmalücken zu beteiligen. Diese Art der Beteiligung ist typisch für Wörterbücher, die als Online-Nachschlagewerke konzipiert sind, wie z.B. das deutsch-englische Wörterbuch LEO Dictionary (Jung/Wimmer 1996) oder der WorldWideWeb Acronym and Abbreviation Server (Flynn o.J.) Sie findet sich aber auch in spaßorientierten Wörterbüchern, z.B. dem sehr liebevoll gemachten schwäbisch-englischen Wörterbuch (Kemmer 1996), in dem unter der Rubrik „missing words“ Lemmalücken bemängelt und englische Übersetzungsvorschläge für Lemmata wie a'mauze, Drucksmulle oder Hegebärlesbauer eingereicht werden können.

3) Beiträge von Spezialisten zu einem bestimmten Wörterbuchgegenstand: Wörterbuchprojekte mit einem relativ spezialisierten Wörterbuchgegenstand nutzen die weltweite Vernetzung des Internet, um andere Spezialisten um Mitwirkung zu bitten. Entsprechende Aufrufe finden sich in akademischen Wörterbuchprojekten wie dem an der Universität Virginia durchgeführten MED-Projekt (Alvarado 1996) oder dem an der Universität Yale angesiedelten Kamusi Projekt, bei dem kollaborativ ein Suaheli-Wörterbuch aufgebaut wird: „The Internet Living Swahili Dictionary is a collaborative work by people all over the world. Together we are working to establish new dictionaries of the Swahili language, both within Swahili and between Swahili and English. We are preparing print-based dictionaries and multi-media computer applications, all accessible to you through this home page. We welcome you, whether you are a beginner or a fluent Swahili speaker." (Benjamin 1995). Vom WWW, ursprünglich ja auch dafür gedacht, den weltweiten Informationsaustausch zwischen hochspezialisierten Wissenschaftern zu erleichtern, erhoffen sich die Initiatoren solcher Wörterbuchprojekte vor allem Qualitätssteigerung und -sicherung, wie das folgende Zitat aus den Paratexten des MED-Projekts zeigt: „One of the advantages the Internet offers over other media of publication is the possibility of collectively authored and edited texts. Such texts are useful for scholary research precisely because of they have been standardized by a community of scholars, and therefore they bear the pedigree of consensus“ (Alvarado 1996).

4) Beliebige Beiträge zu einem vorgegebenen Wörterbuchgegenstand: Nicht um hochwertige Spezialwörterbücher sondern um Spaß am spielerischen Umgang mit Sprache und dem neuen Medium geht es bei Ulkwörterbüchern wie dem „Swedish idioms in painfully literal translation“ (Swedish Idioms), dem „Dorktionary“ (Gaudet/Kleinpeter et al. 1995), oder „The Alternative Dictionaries. international slang“ (Holm o.J.). In diesen Projekten werden alle Wörterbuchartikel von den Internet-Nutzern selbst erstellt; die Initiatoren kümmern sich lediglich um die technische Realisierung. Solche Wörterbücher befinden sich in ständigem Aufbau und steter Veränderung; ein Abschluß ist weder geplant noch erwünscht; der Weg ist das Ziel.

Die in 1) gewünschte Form der Benutzer-Partizipation entspricht bei gedruckten Wörterbüchern ungefähr der Beifügung eines Kommentarzettels mit Bitte um Rücksendung an den Verlag oder den Autor, der die Korrekturen und Anregungen allerdings erst bei der nächsten Auflage berücksichtigen könnte. Die in 2), 3) und 4) beschriebenen Formen der gemeinsamen Wörterbucharbeit sind ohne die Kommunikations- und Informationsdienste des Internet nicht denkbar.

\subsection{Die Verbindung von CD-ROM und Internet}

Im Vergleich zu den auf CD-ROM vertriebenen elektronischen Nachschlagewerken sind die im Internet publizierten Wörterbücher wesentlich schlichter gestaltet und sparsamer mit multimedialen Zusätzen ausgestattet. ${ }^{16}$ Dies liegt u.a. daran, daß Bild-, Ton- und besonders Videoda-

16 Ein typologische Untersuchung zu den im Sommer 1996 verfügbaren WWW-Wörterbücher geben Storrer/ Freese (1997). 
teien sehr umfangreich sind, so daß der großzügige Umgang mit Multimedia-Objekten nicht nur weiter die ohnehin überlastete „Datenautobahn“ verstopft, sondern durch lange Übertragungszeiten - das Akronym WWW wird inzwischen schon durch „weltweites Warten“ aufgelöst - vor allem bei privater Nutzung auch finanziell zu Buche schlägt.

Die Vorteile des Internet - Vielfalt, Aktualität und Kommunikationsdienste - lassen sich jedoch durchaus mit den Vorzügen des offline-Mediums CD-ROM - ansprechendes Design und schneller Zugriff - verbinden, indem von einem auf CD-ROM gespeicherten Nachschlagewerk aus auf ein ergänzendes und stets aktualisiertes Informationsangebot im WWW zugegriffen werden kann.

Diese innovative Idee liegt auch dem Langenscheidt Language Explorer LLE zugrunde, dessen jugendlich-frisch daherkommende Produktinformation sehr vielversprechend klingt:

„Die CD-ROM Surfin' California bietet eine Erlebniswelt rund um das Thema Kalifornien, mit Inhalten zu
den Bereichen Music, Movies, Sports, Hightech, Nature und Melting Pot. Und damit's nicht langweilig
wird, wechseln die Themen wöchentlich. Besonderer Clou der Software sind integrierte Links ins Internet,
mit denen die auf der CD-ROM behandelten Themen jederzeit aktualisiert werden können. (...) Kontakt
und Kommunikation mit internationalen Benutzern werden beim Online-Angebot zu Langenscheidts Lan-
guage Explorer: Surfin' California großgeschrieben. (...). Und wenn's mal Probleme geben sollte, helfen
Euch Links auf ein online-Wörterbuch weiter.“

Was bedeutet es nun aber, daß „die auf der CD-ROM behandelten Themen jederzeit aktualisiert werden können“? Die CD-ROM ist als Datenträger beim momentanen Stand der Technik nicht beschreibbar. Eine „Aktualisierung“ in dem Sinne, daß man sich aktuelle Themen oder etwa ein verbessertes Wörterbuch über eine spezielle Programmfunktion auf die Festplatte seines Rechner laden kann, wird zwar angekündigt, ist aber bislang nicht realisiert; es muß dafür die Speicher-Funktion des Browsers oder ein zusätzliches Programm verwendet werden. Wer sich aber die aktuellen Angebote online anschaut, muß wieder die hohen Ladezeiten und die damit verbundenen Kosten auf sich nehmen. Und diese Kosten entstehen zusätzlich zu den 99 DM, die bereits für die CD-ROM berappt werden mußten. Dieser Preis ist angesichts der bereits in Abschnitt 2 diskutierten Mängel nicht gerechtfertigt, zumal das ,aktualisierte“ Angebot im WWW auch ohne die CD-ROM abgerufen werden kann.

Auch die Hyperlinks zum versprochenen online-Wörterbuch sind auf Anhieb nicht zu finden, jedenfalls nicht in der quicklinks-Liste. Das nicht ganz jugendfreie rap-Lexikon, das zum Zeitpunkt der Abfertigung des Artikels unter dem Themenbereich „music“ angeboten wurde, kann wohl nicht gemeint sein; es ist zur Verbesserung der schulischen Englischnote oder der Kommunikation bei einer Austauschfamilie von eher zweifelhaftem Wert. Vermutlich handelt es sich bei dem angekündigten Wörterbuch um das von Langenscheidt online angebotene zweisprachige online-Wörterbuch (LWE) das zwar tatsächlich im Vergleich mit dem auf CD gebrannten „dictionary“ eine Bereicherung darstellt, sich aber auch nicht ohne Komplikationen auf die eigene Festplatte laden läßt. Da das Internet-Angebot des LLE jedoch großteils in deutscher Sprache verfaßt ist, wird zunächst auch gar kein englisches Wörterbuch mehr benötigt, es sei denn für die reichlich eingestreuten Anglizismen. Insgesamt gilt: Wer von der Leitseite des LLE den Weg zum Wörterbuch findet, der wird im wirklich reichhaltigen weltweiten Angebot ohnehin interessantere Ressourcen finden als die von LLE angebotenen. Neben einem 
stets wachsenden Angebot an Wörterbüchern, ${ }^{17}$ mangelt es auch nicht an englischsprachigen Nachrichtenbretter und on-line Diskussionsgruppen.

Die Nutzung des Internet zum Sprachenlernen ist also grundsätzlich eine gute Idee; die Angebote sind reichhaltig genug und großteils kostenlos verfügbar, ${ }^{18}$ und auch ,elektronische Brieffreundschaften“, sog. e-mail-Tandems, werden auf nicht-kommerzieller Basis für verschiedene Sprachen vermittelt. Bislang fehlt es eher an didaktischen Konzepten und computertechnischer Kompetenz als an geeigneten Hypermedia-Angeboten, um das Internet für den schulischen Deutsch- und Fremdsprachenunterricht nutzbar zu machen. ${ }^{19}$ Eine teure CD-Rom von der Machart des LLE wird dazu jedenfalls nicht benötigt.

\section{Ausblick}

Wenn man Lexikographie nach Wiegand $(1988,733 \mathrm{f}$ ) als wissenschaftliche und kulturelle Praxis begreift, die auf die Entwicklung von Nachschlagewerken hin ausgerichtet ist, dann hatte der Computer in dieser Praxis bislang vornehmlich die Rolle eines Werkzeugs inne, mit dem der lexikographische Arbeitsprozeß beschleunigt und effizienter gestaltet werden kann. Die Hypermedia-Technik und weltweite Computernetzwerke wie das Internet haben den Computer nun zum Medium avancieren lassen, durch das Sprach- und Sachwissen auf neuartige Weise dargestellt und abgerufen werden kann. Dies wird langfristig nicht nur den Prozeß der Wörterbuchherstellung radikal verändern, sondern auch die dabei entstehenden lexikographischen Endprodukte und die Art und Weise, wie diese benutzt werden. Von diesen Entwicklungen werden sowohl Lexikographen als auch Wörterbuchbenutzer profitieren:

Lexikographen von Hypermedia-Wörterbüchern können verschiedene Symbolsysteme nutzen und unterschiedliche Sinnesmodalitäten ansprechen. Multimedia ergänzt das bislang textdominierte Wörterbuch durch Tonobjekte mit Ausspracheangaben und unterstützt durch Bildund Videoobjekte die Bedeutungserläuterung vor allem im Bereich der Nennlexik. Vor allem für die Fachlexikographie ist diese Erweiterung der Darstellungsmittel von großem Nutzen. Im Hypermedia-Wörterbuch besteht nicht länger die Notwendigkeit, durch Abkürzungen und andere standardisierte Textverdichtungsoperationen Druckraum zu sparen. Es können also nicht nur mehr lexikographische Angaben gemacht werden, sondern die Angaben werden durch den Wegfall der Textverdichtungsmittel besser verständlich. Die im Internet angebotenen Kommunikations- und Informationsdienste fördern die rasche und unkomplizierte Kommunikation zwischen Anbietern und Nutzern von Wörterbuchdaten und ermöglichen bislang nicht denkbare Formen der kollaborativen Erarbeitung von Wörterbüchern, die vor allem für die nicht-kommerziell orientierte, wissenschaftliche Lexikographie interessant sind. Die wachsende Zahl von kollaborativ entwickelten Spezialwörterbüchern zeigt, wie im neuen Medium die Grenze zwischen Autoren und Lesern bzw. Benutzern zunehmend verwischt.

17 Wer sich einen aktuellen Überblick über die im WWW verfügbaren Wörterbücher verschaffen möchte, dem sei die umfangreiche, wohlsortierte Sammlung von Robert Beard (Beard 1996) empfohlen.

$18 \mathrm{Vgl}$. Breindl (in Vorbereitung).

19 Zum Einsatz des Internet im Schulunterricht vgl. Wichert (1997) und Döring (1995). 
Für die Wörterbuchbenutzer bietet die neue Publikationsform - Erfahrung im Umgang mit Computern und graphischen Oberflächen vorausgesetzt - einfachere und flexiblere Suchmöglichkeiten zu attraktiv „verpackten“ lexikographischen Angaben. Eine gut gemachte Hypermedia-Anwendung kann nicht nur den Zugriff auf das lexikalische Wissen beschleunigen, sondern auch - im Sinne des Grimmschen Lesebuchgedankens - zum Herumstreifen im lexikalischen Informationsraum anregen. Mit interaktiven Werkzeugen können die dabei gefundenen „Wortschätze“ nach individuellen Interessen zusammengestellt, kommentiert, verschlagwortet und weiter bearbeitet werden, der Benutzer kann das Wörterbuch an seine Bedürfnisse anpassen und mitgestalten.

Lexikographische Hypermedia-Anwendungen erweitern auch den Gegenstandsbereich der wissenschaftlichen Wörterbuchforschung und zwar in verschiedenen Teilgebieten. Wie in Abschnitt drei gezeigt wurde, basieren die meisten Angebote bislang auf sehr grobkörnig segmentierten Printwörterbüchern, deren Segmente über rein stringorientiertere automatische Verknüfungsmechanismen verbunden sind. Metalexikographische Beschreibungen von Strukturen gedruckter Wörterbuchtexte können zu einer feinkörnigeren Segmentation nach funktionalen Kriterien beitragen; die Lexikologie kann die Kategorien und Prinzipien beisteuern, nach denen die entstehenden Textsegmente durch Verknüpfungen relationiert werden. Auf der Basis bisheriger Forschungen sollte die Wörterbuchkritik Kriterien und Standards für die Qualitätsbeurteilung elektronischer Wörterbücher entwickeln. Gerade weil der Inhalt einer CD-ROM - anders als beim Buch - häufig vor dem Kauf nicht eingesehen werden kann, werden momentan viele Produkte von zweifelhafter Qualität vertrieben. Wie Neth/Müller im Fazit ihrer Rezension zu deutsch-englischen Wörterbüchern zurecht schreiben, kann man „mühelos ein völlig untaugliches Werk erwerben. In der Begeisterung über die Möglichkeiten des Mediums CDROM haben viele Autoren den Sinn und Zweck eines Wörterbuchs bisweilen vergessen." (Neth/Müller 1997, 138). Die Beurteilung der Wörterbücher läßt sich untermauern durch empirische Untersuchungen zur Wörterbuchbenutzung, die im elektronischen Medium durch Programme unterstützt werden können, die alle Eingaben des Benutzers protokollieren und deren „Lesewege“ aufzeichnen. Die dabei gewonnenen Ergebnisse sind sicher zum Teil auch für die Printlexikographie nützlich, die es mit Sicherheit auf absehbare Zeit weiterhin geben wird und geben sollte. Um verschiedene Publikationsformen im Hinblick auf ihre Stärken für die lexikographische Sprachbeschreibung miteinander zu vergleichen und um die Erstellung qualitativer Endprodukte im gedruckten und elektronischen Medium durch wissenschaftliche Forschungen zu unterstützen, bedarf es nicht einer eigenständigen Metalexikographie für elektronische Nachschlagewerke; wünschenswert wäre vielmehr eine Ausweitung des Gegenstandsbereichs in allen metalexikographischen Teilbereichen.

\section{Literatur}

Alvarado, R. C. (1996): The Mayan Epigraphic Database Project. Online. Internet. 28.07.1997. Available http://jefferson.village.virginia.edu/med/home.html.

ASL: A Basic Dictionary of ASL Terms. Online. Internet. Available http://home.earthlink.net/ masterstek/ ASLDict.html.

Beard, R. (1996): A Web of On-Line Dictionaries. Online. Internet. 14.07.1997. Available http://www.bucknell. edu/ rbeard/diction.html. 
Benjamin, M. (1995): Swahili - The Kamusi Project. Online. Internet. 02.03.1997. Available http://www.cis. yale.edu:80/swahili/

Bee-Book (nR) (1996): Die neue deutsche Rechtschreibung. München. CD-ROM.

Bee-Book (TWF) (1996): Taschenwörterbuch Französisch-Deutsch. Gütersloh. CD-ROM. München.

Bläsi, C.; Koch, H.-D. (1992): „Maschinelle Strukturerschließung von Wörterbuchartikeln mit Standardmethoden.“ In: Lexikographica 7 (1991) (1992), 182-201.

Bogaschewsky, R. W. (1992): „Hypertext-/Hypermedia-Systeme. Ein Überblick.“ In: Informatik-Spektrum 15/3 (1992), 127-143.

Bookshelf (1995): Microsoft Bookshelf '95. Multimedia Reference Library. Microsoft. CD-ROM.

Breidt, E. (im selben Band): „Neuartige Wörterbücher für Mensch und Maschine: Wörterbuchdatenbanken in COMPASS.“

Breindl, E. (in Vorbereitung): „DaF goes Internet. Neuere Entwicklungen im Bereich Deutsch als Fremdsprache." In: Deutsche Sprache (in Vorbereitung).

Cailliau, R. (1995): A Little History of the World Wide Web. Online. Internet. 03.10.1995. Available http:// www.w3.org/pub/WWW/History.html.

Cölfen, E. (o.J.): Rezension zu: OED - Oxford English Dictionary $2^{\text {nd }}$ edition CD-ROM. Online. Internet. Available http://www.linse.uni-essen.de/reviews/software/oed.htm.

Cölfen, E.; Cölfen, H.; Schmitz, U. (1997): Linguistik im Internet. Das Buch zum Netz. Opladen.

Döring, N. (1995): „Internet: Bildungsreise auf der Infobahn.“ In: Issing, L. J.; Klimsa, P. (Hgg.) (1995): Information und Lernen mit Multimedia. Weinheim, 221-240.

DUDEN: Duden. Die deutsche Rechtschreibung. Mannheim/Leipzig/Wien/Zürich. CD-ROM.

Flynn, P. (o.J.): The World Wide Web Acronym and Abbreviation Server. Online. Internet. Available http:// www.ucc.ie/info/net/acronyms/acro.html

Freisler, S. (1994): „Hypertext - eine Begriffsbestimmung.“ In: Deutsche Sprache 22 (1994), 19-50.

Gaudet, M. P.; Kleinpeter, J. L.; Eunice, W. C. (1995): Dorktionary. Online. Internet. 06.08.1997. Available http://www.latech.edu/ jlk/jwz/dorctionary/index.shtml

Gloor, P. A. (1990): Hypermedia-Anwendungsentwicklung. Eine Einführung mit HyperCard-Beispielen. Stuttgart.

Grimm, J. (1854): „Vorrede zum Deutschen Wörterbuch.“ In: Grimm, J.; Grimm, W. (1854): Deutsches Wörterbuch. Leipzig, I-LXVIII.

Haak, J. (1995): „Interaktivität als Kennzeichen von Multimedia und Hypermedia.“ In: Issing, L. J.; Klimsa, P. (Hgg.) (1995): Information und Lernen mit Multimedia. Weinheim.

Hauser, R.; Storrer, A. (1994): „Dictionary Entry Using the LexParse System.“ In: Lexicographica 9 (1994), 174-219.

Hauser, R.; Storrer, A. (1996): „Probleme und Lösungen beim Parsen von Wörterbüchern.“ In: Feldweg, H.; Hinrichs, E. (Hgg.) (1996): Lexikon und Text. Wiederverwendbare Methoden und Ressourcen zur linguistischen Erschließung des Deutschen. Tübingen, 61-77.

Hausmann, F. J.; Reichmann, O.; Wiegand, H. E. et al. (Hgg.) (1990): Wörterbücher. Ein internationales Handbuch zur Lexikographie, 2. Teilband. Berlin/New York.

Hausmann, F. J.; Wiegand, H. E. (1989): „Component Parts and Structures of Monolingual Dictionaries: A Survey.“ In: Hausmann, F. J.; Reichmann, O.; Wiegand, H. E. et al. (Hgg.) (1989): Wörterbücher. Ein internationales Handbuch zur Lexikographie, 1. Teilband. Berlin/New York, 328-360.

Hausser, R. (Hg.) (1996): Linguistische Verifikation. Dokumentation zur Ersten Morpholympics 1994. Tübingen.

Herring, S. C. (Hg.) (1996): Computer-Mediated Communication. Linguistic, social and cross-cultural perspectives. Amsterdam, Philadelphia.

Holm, H.-C. (o. J.): The Alternative Dictionaries. International Slang - An Internet Collaborative Project. Online. Internet. Available http://www.notam.uio.no/ hcholm/altlang/

Jung, A.; Wimmer, P. K. (1996): LEO Dictionary: German <-> English. Online. Internet. 09.06.1997. Available http://www.leo.org./cgi-bin/dict-search

Kammerer, M. (1997): „Hypertextualisierung gedruckter Wörterbuchtexte: Verweisstrukturen und Hyperlinks. Eine Analyse anhand des Frühneuhochdeutschen Wörterbuches.“ In: Storrer, A.; Harriehausen, B. (Hgg.) (1997): Hypermedia für Lexikon und Grammatik. Tübingen.

Kemmer, T. (1996): Swabian into English. Online. Internet. 19.06.1997. Available http://www.architektur.unistuttgart.de:1200/users/tk/sw/swab.html.

Kretschmer, B. (1996): Das Internet Dschungelbuch. Düsseldorf. 
Kuhlen, R. (1991): Hypertext. Ein nicht-lineares Medium zwischen Buch und Wissensbank. Berlin/Heidelberg.

Leckebusch, J. (1995): „Übersetze! Sieben englische Wörterbücher im Vergleich.“ In: c't 1995/11 (1995), 246252.

Lemberg, I.; Petzold, S.; Speer, H. (im selben Band): „Der Weg des Deutschen Rechtswörterbuchs in das Internet."

Lenke, N.; Schmitz, P. (1995): „Geschwätz im 'Globalen Dorf’ - Kommunikation im Internet.“ In: OBST 50 (1995), 117-141.

LIED (1993): Longman Interactive English Dictionary. CD-ROM.

LLE (1997): Langenscheidt's Language Explorer. Berlin/München. CD-ROM.

LWE: Langenscheidt Wörterbuch Englisch. Online. Internet. Available http://www.langenscheidt.de/dictio/ dictio.htm.

Neth, H.; Müller, T. (1997): „Angloviel. 25 Englischwörterbücher für Windows und Mac.“ In: c't 1997/1 (1997), 116-138.

netscape (1997): Netscape Homepage. Online. Internet. Available http://home.netscape.com.

Nielsen, J. (1995): Multimedia and Hypertext. The Internet and Beyond. Boston.

OED: Oxford English Dictionary. Oxford. CD-ROM.

PC-Bib (DUW/DOG) (1989/1990): Deutsches Universal Wörterbuch A-Z/DUDEN OXFORD Großwörterbuch Englisch. Mannheim/Leipzig/Wien/Zürich. CD-ROM.

Raymond, D. R.; Tompa, F. W. M. (1988): „Hypertext and the Oxford English Dictionary.“ In: Communications of the $A C M 31 / 7$ (1988).

Riehm, U. W., Bernd (1995): Multimedia. Mythen, Chancen und Herausforderungen. Mannheim.

Schulmeister, R. (1993): Dictionary for Computer Terms with Signs. Hamburg.

- (1996): Grundlagen hypermedialer Lernsysteme. Theorie - Didaktik - Design. Bonn et al.

Schult, T. J. (1997): „Darf's eine Scheibe mehr sein? Nachschlagen perfekt: die CD-ROM erobert den Alltag.“ In: $c^{\prime} t$ 1997/1 (1997), 115.

Storrer, A. (1996): „Metalexikographische Methoden in der Computerlexikographie.“ In: Wiegand, H. E. (Hg.) (1996): Wörterbucher in der Diskussion II. Tübingen, 239-255.

- (1996): „Wörterbücher zum Anklicken. Ein kleiner Rundgang durch die PC-Bibliothek.“ In: Sprachreport 2/95 (1996), 9-10.

Storrer, A./Freese, K. (1997): „Wörterbücher im Internet.“ In: Deutsche Sprache 1996/2 (1997), 97-153.

Swedish Idioms: Swedish Idioms in painfully literal translation. Online. Internet. 12.02.1996. Available http:// www.webcom.com/eha/idiom.html

Thielen, C./Breidt, E./Feldweg, H. (1997): „COMPASS - Ein intelligentes Wörterbuchsystem für das Lesen fremdsprachiger Texte." In: Storrer, A.; Harriehausen, B. (Hgg.): Hypermedia für Lexikon und Grammatik. Tübingen.

Todesco, R. (1995): „Schränkt Hypertext die Sprache ein? Ein Erfahrungsbericht über den Versuch, ein HyperLexikon zu schreiben." In: OBST 50 (1995), 165-176.

Weidenmann, B. (1995): „Multikodierung und Multimodalität im Lernprozeß.“ In: Issing, L., J.; Klimsa, P. (Hgg.) (1995): Information und Lernen mit Multimedia. Weinheim, 65-84.

Wichert, A. (1997): „Hypertext im Deutschunterricht. Überlegungen zur Rhetorik und Didaktik des Hypertexts." In: OBST 55 (1997), 118-131.

Wiegand, H. E. (1983): „Ansätze zu einer allgemeinen Theorie der Lexikographie.“ In: Schildt, J.; Viehweger, D. (Hgg.) (1983): Die Lexikographie von heute und das Wörterbuch von morgen. Analysen - Probleme Vorschlage. Berlin, 92-127.

- (1987): „Wörterbuchartikel als Text.“ In: Harras, G. (Hg.) (1987): Das Wörterbuch. Artikel und Verweisstrukturen. Düsseldorf, 30-120.

- (1988): „Was ist eigentlich Fachlexikographie? Mit Hinweisen zum Verhältnis von sprachlichem und enzyklopädischem Wissen.“ In: Munske, H. H.; von Polenz, P.; Reichmann, O. et al. (Hgg.) (1988): Deutscher Wortschatz: Lexikologische Studien: Ludwig Erich Schmitt zum 80. Geburtstag von seinen Marburger Schülern. Berlin/New York, 729-790.

- (1989): „Aspekte der Makrostruktur im allgemeinen einsprachigen Wörterbuch: alphabetische Anordnungsformen und ihre Probleme." In: Hausmann, F. J.; Reichmann, O.; Wiegand, H. E. et al. (Hgg.) (1989): Wörterbücher. Ein internationales Handbuch zur Lexikographie, 1. Teilband. Berlin/New York, 371-409. 
- (1989): „Der Begriff der Mikrostruktur: Geschichte, Probleme, Perspektiven.“ In: Hausmann, F. J.; Reichmann, O.; Wiegand, H. E. et al. (Hgg.) (1989): Wörterbücher. Ein internationales Handbuch zur Lexikographie, 1. Teilband. Berlin/New York, 409-462.

- (1991): „Printed Dictionaries and their Parts as Text. An Overview of more Research as an Introduction.“ In: Lexikographica 6 (1990) (1991), 1-124.

- (1996): „Textual Condensation in Printed Dictionaries. A Theoretical Draft.“ In: Lexikos 6 (1996), 133158.

- (1996): „Über die Mediostrukturen bei gedruckten Wörterbüchern.“ In: Zettersten, A.; Hjornager Pedersen, V. (Hgg.) (1996): Symposium on Lexicography May 5-6 1994 at the University of Copenhagen. Tübingen, $11-43$.

- (1997): „Printed language dictionaries and their standardization: Notes on the progress toward a general theory of lexicography." In: Hock, H. H. (Hg.) (1997): Historical, Indo-European, and Lexicographical Studies. A Festschrift for Ladislav Zgusta on the Occasion of his $70^{\text {th }}$ Birthday. Berlin/New York, 319393.

- (im selben Band): „Neues und Altes zu den Makrostrukturen.“

WWLIA (1995): The WWLIA Legal Dictionary. Online. Internet. Available http://www.islandnet.com/ wwlia/ diction.htm. 